\title{
Evidence for eight node mixed-symmetry superconductivity in a correlated organic metal
}

\author{
Daniel Guterding, ${ }^{1}$ Sandra Diehl, ${ }^{2,3}$ Michaela Altmeyer, ${ }^{1}$ Torsten Methfessel, ${ }^{3}$ \\ Ulrich Tutsch, ${ }^{4}$ Harald Schubert ${ }^{4}$ Michael Lang, ${ }^{4}$ Jens Müller, ${ }^{4}$ Michael Huth, ${ }^{4}$ \\ Harald O. Jeschke, ${ }^{1}$ Roser Valentí, ${ }^{1}$ Martin Jourdan, ${ }^{3}$ and Hans-Joachim Elmers ${ }^{3}$, * \\ ${ }^{1}$ Institut für Theoretische Physik, Goethe-Universität Frankfurt, \\ Max-von-Laue-Str. 1, 60438 Frankfurt am Main, Germany \\ ${ }^{2}$ Graduate School Materials Science in Mainz, Staudingerweg 9, 55128 Mainz, Germany \\ ${ }^{3}$ Institut für Physik, Johannes Gutenberg-Universität Mainz, Staudingerweg 7, 55128 Mainz, Germany \\ ${ }^{4}$ Physikalisches Institut, Goethe-Universität Frankfurt, \\ Max-von-Laue-Str. 1, 60438 Frankfurt am Main, Germany
}

(Dated: September 11, 2018)

\begin{abstract}
We report a combined theoretical and experimental investigation of the superconducting state in the quasi-two-dimensional organic superconductor $\kappa-(\mathrm{ET})_{2} \mathrm{Cu}\left[\mathrm{N}(\mathrm{CN})_{2}\right] \mathrm{Br}$. Applying spinfluctuation theory to a low-energy material-specific Hamiltonian derived from ab initio density functional theory we calculate the quasiparticle density of states in the superconducting state. We find a distinct three-peak structure that results from a strongly anisotropic mixed-symmetry superconducting gap with eight nodes and twofold rotational symmetry. This theoretical prediction is supported by low-temperature scanning tunneling spectroscopy on in situ cleaved single crystals of $\kappa$-(ET) $)_{2} \mathrm{Cu}\left[\mathrm{N}(\mathrm{CN})_{2}\right] \mathrm{Br}$ with the tunneling direction parallel to the layered structure.
\end{abstract}

PACS numbers: $74.25 . \mathrm{Jb}, 74.50 .+\mathrm{r}, 74.70 . \mathrm{Kn}, 74.20 . \mathrm{Pq}$

Introduction.- It has been widely accepted that $\kappa$ $(\mathrm{ET})_{2} X$ organic charge-transfer salts [where ET denotes bis(ethylenedithio)tetrathiafulvalene and $X$ a monovalent anion] share essential features with cuprates regarding their superconducting state [1]. In both classes of materials the electronic structure is quasi-two-dimensional and superconductivity emerges in the vicinity of an antiferromagnetically ordered Mott insulating phase. The transformation of these Mott insulators into superconductors can be achieved either by doping (cuprates) or by increasing the bandwidth (organics) through the application of physical or chemically-induced pressure, see e.g. Ref. 2, In view of these similarities it is natural to investigate whether a related coupling mechanism is operative in both classes of materials.

A direct determination of the pairing mechanism is extremely difficult, if not impossible, and therefore most efforts have been directed towards the determination of the symmetry of the superconducting energy gap function. For the cuprates, thanks to the availability of phasesensitive probes, compelling evidence was provided early on that the gap has predominantly $d_{x^{2}-y^{2}}$ symmetry 3 , 4. The lack of such probes for the organic charge-transfer salts, rooted in difficulties in proper material preparation, makes the situation less clear. There has been a longlasting controversy about the gap symmetry in the most widely studied materials $\kappa$ - $(\mathrm{ET})_{2} \mathrm{Cu}\left[\mathrm{N}(\mathrm{CN})_{2}\right] \mathrm{Br}$ (in short $\kappa$ - $\mathrm{Br})$ and $\kappa$-(ET) $)_{2} \mathrm{Cu}(\mathrm{NCS})_{2}(\kappa$-NCS) with some results in support of an $s$-wave and others consistent with a $d$ wave scenario (see the reviews [2, 5, 7] for the status of the discussion up to the year 2006). More recent attempts include magnetocalorimetry [8, surface impedance [9] and high-resolution specific heat measurements [10, 11, both in favor of $d$-wave pairing, even though earlier results of the specific heat favored $s$-wave symmetry [1214. On the other hand, by analyzing measurements of elastic constants [15] a mixed order parameter of either $A_{1 g}+B_{1 g}$ or $B_{2 g}+B_{3 g}$ has been claimed for $\kappa$ - $\mathrm{Br}$ following the classification of irreducible representations of the material's orthorhombic $D_{2 h}$ point group.

In the above-mentioned studies the gap function could be determined only indirectly from the temperature dependence of the quantity investigated. In contrast, a direct examination of the energy gap and its angular dependence is possible, in principle, through scanning tunneling spectroscopy (STS). Such STS studies have been performed by tunneling either into as grown surfaces for $\kappa$-NCS [16] and $\kappa$-Br [17, or into focused-ion-beam-cut surfaces for partially deuterated $\kappa$-Br [18. The results were found to be consistent with a $d$-wave order parameter. However, with the exception of Ref. 18, no surface characterization was provided in these studies.

In this Letter we report evidence for an anisotropic superconducting gap structure for $\kappa$-Br based on a combined theoretical and STS investigation. By first constructing a two-dimensional Hubbard model of the ET layer from ab initio density functional theory (DFT) calculations and subsequently applying spin-fluctuation theory to this model, we predict the presence of a strongly anisotropic superconducting gap. As a consequence of the orthorhombicity of the crystal structure and the resulting details of the electronic structure the order parameter does not have pure $d$-wave symmetry. Instead, an eight node twofold rotationally symmetric state is 
realized, which we identify as (extended) $s+d_{x^{2}-y^{2}}$. This pairing symmetry results in a quasiparticle density of states (DOS) with a distinct three-peak structure. STS measurements performed on well-defined surfaces obtained from a recently developed in situ cleaving technique [19] support this theoretical finding.

Theory.- We performed ab initio density functional theory (DFT) calculations for $\kappa$-Br within an all-electron full-potential local orbital (FPLO) [20] basis and subsequently derived a tight-binding model from projective molecular orbital Wannier functions [21, 22]. The resulting low-energy Hamiltonian $H_{0}=\sum_{i j} t_{i j}\left(c_{i}^{\dagger} c_{j}+\right.$ h.c. $)$, with hopping parameters $t_{i j}$ between ET molecules at sites $i$ and $j$, consists of four bands arising from the highest occupied molecular orbitals of the ET molecules in the crystallographic unit cell and is 3/4-filled (see Ref. 22).

Previous theoretical approaches [23] approximated the real crystal structure by an anisotropic triangular lattice of $(\mathrm{ET})_{2}$ dimers [24]. This approximation was shown to be justified in the insulating phase of $\kappa$-(ET) ${ }_{2} X$ materials [25], but not in the superconducting state [26]. Hence our method, which is based on an ab initio derived Hamiltonian with the full symmetry of the ET layer, is more realistic than previous approaches.

In the following we take the rigorous viewpoint that superconductivity in $\kappa$ - $\mathrm{Br}$ is solely driven by electronelectron interactions, see e.g. 2, 27, 28. Therefore, we add an intramolecular Hubbard interaction term $H_{\text {int }}=$ $U \sum_{i} n_{i \uparrow} n_{i \downarrow}$ to $H_{0}$. In this setup we calculate the optimal geometry of the superconducting gap function using random phase approximation (RPA) spin-fluctuation theory in the spin-singlet channel 29. For computational details see Ref. 30. We stress, however, that our results do not exclude a contribution from electron-phonon interactions, indications of which have been found, e.g., in studies of the isotope effect [31] and phonon renormalization 32.

From the RPA calculation we find that the superconducting order parameter has eight nodes [see Fig. 11(a)] in contrast to previous reports of pure $d$-wave states with four nodes [6, 23]. The first set of nodes labelled $\alpha$ in Fig. 1(a) appears on the quasi-one-dimensional part of the Fermi surface. The four nodes on the elliptic part of the Fermi surface, denoted $\beta$ in Fig. 1 (a), are close to the Brillouin zone diagonals. The full angular dependence of the gap function is shown in Fig. 1 (b). The angles corresponding to the node positions are $\varphi_{\alpha} \sim 15^{\circ}$ and $\varphi_{\beta} \sim 58^{\circ}$, all measured with respect to the $k_{x}$-direction. Nodes at other angles are symmetry equivalent to $\alpha$ and $\beta$. The three maxima of the superconducting gap magnitude are indicated by letters $\mathrm{A}, \mathrm{B}$ and $\mathrm{C}$ in Fig. 1 . The corresponding angles are $\varphi_{A}=90^{\circ}, \varphi_{B}=45^{\circ}$ and $\varphi_{C}=0^{\circ}$. Maxima at other angles are symmetry equivalent to $\mathrm{A}, \mathrm{B}$ and $\mathrm{C}$.

The quasiparticle DOS $\rho_{q p}(E)$ in the superconducting state can be calculated from the momentum structure of (a)
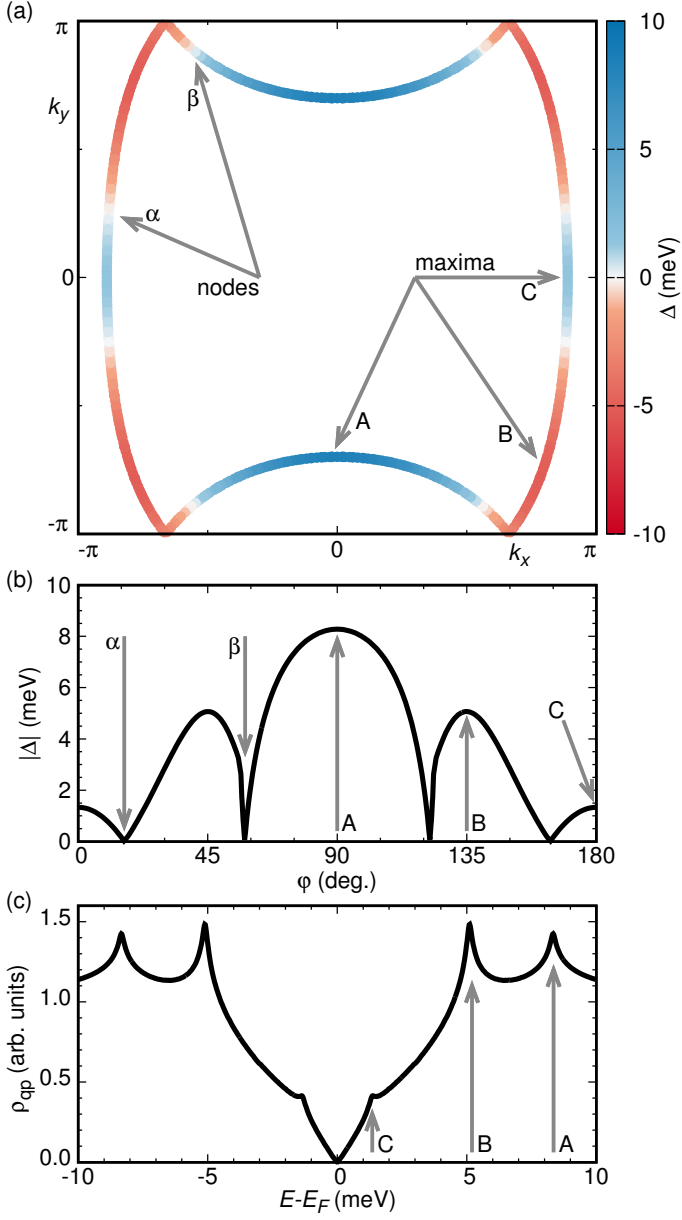

FIG. 1. (Color online) Ab initio calculated results for (a) superconducting gap $\Delta$ on the Fermi surface, (b) absolute value $|\Delta|$ of the superconducting gap as a function of the angle $\varphi$ in the $k_{x}-k_{y}$-plane measured with respect to the $k_{x}$-direction and (c) quasiparticle DOS $\rho_{q p}$ in the superconducting state of $\kappa$-Br. The maxima of the superconducting gap magnitude are indicated by arrows A, B and C, while the positions of nodes are indicated by arrows $\alpha$ and $\beta$. The energy scale is set to $\Delta_{0}=10 \mathrm{meV}$. A small broadening of $\Gamma=0.07 \mathrm{meV}$ is included, here.

the superconducting gap $\Delta(\vec{k})$, as given in Eq.11. A small broadening of the energy spectrum due to finite quasiparticle lifetimes is modeled by the parameter $\Gamma[33$.

$$
\rho_{q p}(E, \Gamma) \propto \sum_{\vec{k}} \operatorname{Re} \frac{|E+i \Gamma|}{\sqrt{(E+i \Gamma)^{2}-\Delta(\vec{k})^{2}}}
$$

The main difference between our formulation (Eq. 1) and conventional approaches [16 18] is that we do not assume the Fermi surface to be a concentric circle. Instead we transform the usual angular integration (see Refs. 1618, and 30) into a sum over the discretized ab initio Fermi surface. Our approach can therefore discriminate between different $d$-wave solutions already on the level of the quasiparticle DOS. The approximations made in the 
derivation of Eq. 1 are detailed in Ref. 30. The quasiparticle DOS calculated from Eq. 1, using the gap calculated from RPA for $\Delta(\vec{k})$, shows three distinct features [Fig. 1.(c)] that correspond to the gap maxima A, B and $\mathrm{C}$ already discussed.

Experiment.- Single crystals of $\kappa$-Br were grown in an electrochemical crystallization process [34]. All investigations were performed with a commercial low-temperature scanning tunneling microscope (STM) under ultra-high vacuum (UHV) conditions with a base pressure of about $5 \cdot 10^{-11}$ mbar. In order to obtain a clean surface as is essential for high-resolution STM investigations, we cleaved the crystal surface with a homebuilt cutter in UHV. After in situ cleaving, the sample was mounted into the precooled STM stage. Measurements have been performed on three single crystals, yielding essentially identical results.

By this procedure, the cooling rate of the sample, relevant at the glass-like ethylene-endgroup ordering transition $T_{g}$, is estimated to be about $-1 \mathrm{~K} / \mathrm{min}$ at $T_{g} \approx$ 75-80 K (see Ref. 35). This implies a residual disorder in the orientational degrees of freedom of the ET molecules' terminal ethylene groups of order $3 \%$ 36, 37. For details on the STM/STS measurements, see Ref. 19 .

Fig. 2(a) shows a $(30 \times 30) \mathrm{nm}^{2}$ STM image of a $\kappa$-Br crystal cleaved perpendicular to the molecular layers (for the parallel topography see Refs. 19] and 38). The STM image reveals a stripe pattern with an average width of $29.9 \AA$ [see Fig. 2(b)] which is in good agreement with the lattice constant of $\kappa$ - $\mathrm{Br}$ in the $c$-direction. One period of the stripe pattern consists of two ET and two anion layers. For a better illustration Fig. 2(b) shows the height profile along the white shaded area in (a), wherein the position of the anion layers is marked by red stripes. The height profile is mainly a mapping of the topographical surface profile, but is also influenced by the local density of states. By analyzing the height profile along a single unit cell we conclude that the cutting plane is rotated by $\varphi_{a b}=60^{\circ}$ about the $c$-axis with respect to the $b$-axis (see Ref. 30).

Next, we measured the differential conductance $\mathrm{d} I / \mathrm{d} V$ of the ET layer and the anion layer in the temperature range from $5 \mathrm{~K}$ to $13 \mathrm{~K}$ [see Fig. 2(c) and (d) for the data at $5 \mathrm{~K}]$ in order to extract information about their DOS. The $\mathrm{d} I / \mathrm{d} V$ spectra have been normalized to the tunneling transmission function $T(V)$, which is almost linear in the measured energy range and accounts for a small asymmetry observed in the original spectra [39. Assuming a constant DOS for the tip material, $(\mathrm{d} I / \mathrm{d} V) / T(V)$ is proportional to the differential conductance $D(V)$ of the sample.

The differential conductance is non-zero at $E=E_{\mathrm{F}}$ for the spectrum measured parallel to the ET layer [Fig. 2(c)]. In contrast, the differential conductance spectrum measured at the anion layer [Fig. 2(d)] approaches zero at the Fermi edge $\left[\mathrm{d} I / \mathrm{d} V\left(E=E_{F}\right) \approx 0\right]$ indi-
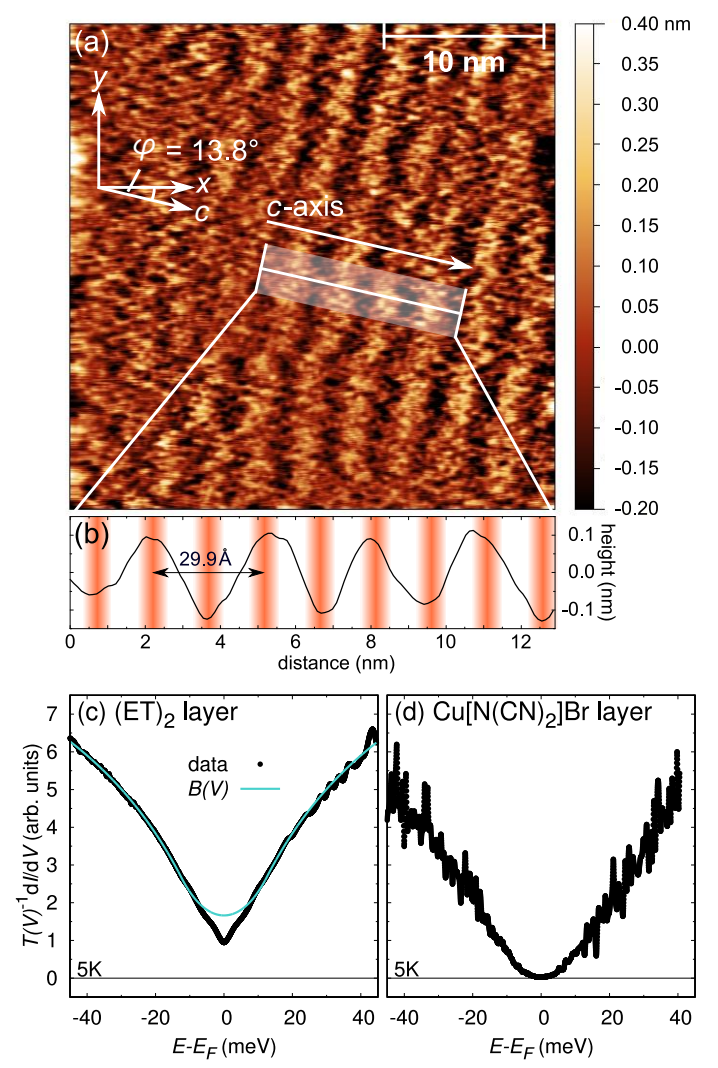

FIG. 2. (Color online) (a) $(30 \times 30) \mathrm{nm}^{2}$ STM image of the crystal surface with tunneling direction parallel to the layered structure of the $\kappa$-Br crystal revealing a stripe pattern $\left(T=5 \mathrm{~K}, I=60 \mathrm{pA}, U_{\mathrm{tip}}=30 \mathrm{mV}\right)$. (b) The height profile along the marked area in (a) reveals an average stripe width of $29.9 \AA$. The red stripes indicate the position of the insulating layers. (c) Conductance spectra $\mathrm{d} I / \mathrm{d} V / T(V)$ at $T=5 \mathrm{~K}$ (black dots) measured at the ET layer and (d) the $\mathrm{Cu}\left[\mathrm{N}(\mathrm{CN})_{2}\right] \mathrm{Br}$ layer with $-e U_{\text {tip }}=e V=E-E_{F}$. The function $B(V)$ is also plotted in (c) (see text for discussion).

cating the expected insulating behavior. The $V$-shaped feature for $|e V|>15 \mathrm{meV}$ results from a logarithmic suppression of the density of states at the Fermi edge described by a function $B(V)$ [see Fig. 2(c)] which has been assigned to electronic disorder [19]. Temperaturedependent measurements up to $13 \mathrm{~K}$ (see Ref. [30) show a nearly temperature-independent function for the DOS $B(V)$ as described by Refs. 19 and 40 .

An important piece of information is obtained from the second derivative of $\mathrm{d} I / \mathrm{d} V$ w.r.t. the voltage as presented in Fig. 3(a). This function at $T=5 \mathrm{~K}$ shows three pairs of narrow minima (denoted as A, B and C), which correspond to superconducting coherence peaks and will be discussed further below. The pronounced maximum at $E_{F}$ reflects the zero-bias anomaly of the normal-state DOS [19].

The conductance spectrum related to the superconducting phase is given by $S(V)=[B(V) T(V)]^{-1} d I / d V$. In Fig. 3(b), $S(V)$ measured parallel to the layered crys- 
TABLE I. Values of the parameters in Eq. 2 obtained by mapping the calculated $S(V)$ to the experimental spectra.

\begin{tabular}{ccccccc}
\hline \hline$T(\mathrm{~K})$ & $c_{s_{1}}$ & $c_{d_{1}}$ & $c_{s_{2}}$ & $\Delta_{0}(\mathrm{meV})$ & $\Gamma(\mathrm{meV})$ & $\mathrm{x}$ \\
\hline 5 & -0.109 & -0.276 & -0.615 & 12.218 & 0.690 & 0.520 \\
7 & -0.128 & -0.280 & -0.592 & 10.638 & 0.641 & 0.603 \\
9 & -0.064 & -0.317 & -0.620 & 7.376 & 0.000 & 0.466 \\
11 & -0.158 & -0.200 & -0.642 & 2.984 & 0.035 & 0.188 \\
\hline \hline
\end{tabular}

tal structure is plotted for temperatures between $5 \mathrm{~K}$ and $13 \mathrm{~K}$. The two main features in the second derivative [shown by arrows A and B in Fig. 3(b)] are already visible in the bare conductance spectra, whereas feature $\mathrm{C}$ becomes discernible only in the second derivative of the spectra.

Discussion.- Our theoretical calculations predict an anisotropic superconducting order parameter. We note that a very accurate representation of the calculated gap on the Fermi surface is given in terms of extended $s$ - and $d$-wave functions (see also Ref. 30): $\Delta(\vec{k})=$ $\Delta_{0}\left[c_{s_{1}}\left(\cos k_{x}+\cos k_{y}\right)+c_{d_{1}}\left(\cos k_{x}-\cos k_{y}\right)+c_{s_{2}}\left(\cos k_{x}\right.\right.$. $\left.\cos k_{y}\right)$ ]. Although our experiments resolve the existence of feature $\mathrm{C}$ only in the second derivative of the conductance, the shape of the experimental tunneling spectra [Fig. 3(b)] bears the essential features predicted by our theory [Fig. 1(c)]. Features A and B are only slightly shifted with respect to each other.

In order to connect the calculated quasiparticle DOS (Eq. 1) to the measured spectrum $S(V)$ we investigate whether quantitative agreement can be reached by finetuning some parameters. In this process the symmetry of the superconducting state is kept fixed to the theoretical prediction (mixed extended $s+d_{x^{2}-y^{2}}$ ). We calculate $S(V)$ from Eq. 2 (see Ref. 30), where $f(E)$ is the Fermi function, $V$ is the bias voltage and $x$ is a background shift, which accounts for parasitic conduction paths.

$$
\begin{aligned}
S(V) & =\frac{1}{B(V) T(V)} \frac{d I(V)}{d V} \\
& \propto \int_{-\infty}^{\infty} d E\left[\rho_{q p}(E)(1-x)+x\right] \frac{-d f(E+e V)}{d V}
\end{aligned}
$$

Using the expression for $\Delta(\vec{k})$ given above, we reevaluate Eqs. 1 and 2 with parameter sets $\left\{\Delta_{0}, c_{s_{1}}, c_{d_{1}}\right.$, $\left.c_{s_{2}}, x, \Gamma\right\}$ until optimal agreement with the experimental spectra in the interval $[-12,+12] \mathrm{meV}$ is reached. The corresponding calculated spectra are shown in Fig. 3(b) as solid red lines. The optimal parameter values listed in Table 1 are consistent throughout the investigated parameter range. Only the origin of the non-monotonous behavior of $\Gamma$ is currently unclear.

The nodal positions we find in our study are particularly appealing because of the ongoing controversy about the realization of either $d_{x y}$ and $d_{x^{2}-y^{2}}$ pairing in quasi-2D organic superconductors. In previous works, a fourfold rotational symmetry constraint was introduced
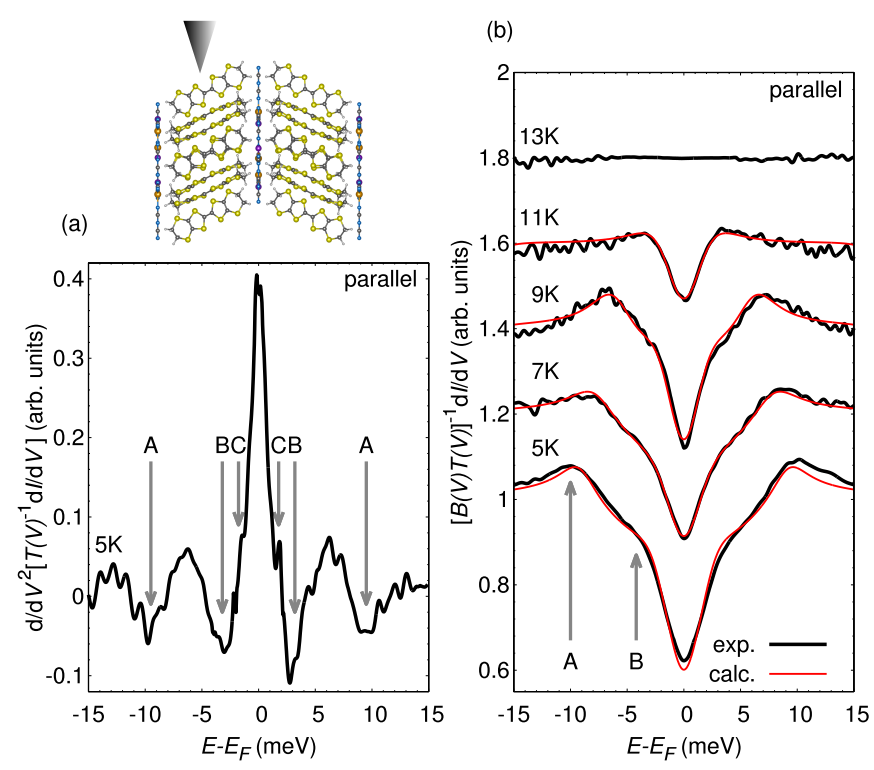

FIG. 3. (Color online) (a) Second derivative of the conductance spectrum $\mathrm{d} I / \mathrm{d} V / T(V)$ at $T=5 \mathrm{~K}$ shown in Fig. 22(c). Arrows labeled by A, B and $\mathrm{C}$ indicate three pairs of minima which are symmetric to the origin, corresponding to the coherence peaks partially seen in (b). (b) Conductance spectra $S(V)=1 /[B(V) T(V)] d I / d V$ of the superconducting state as a function of $e V=E-E_{F}$ at different temperatures measured parallel to the layered crystal structure. The red lines show mappings of Eq. (2) to the measured data.

based on single-band Hubbard-type models proposed for these systems with a dimer of molecules per site on an anisotropic triangular lattice [23, 25, 41,43]. Inspired by recent $a b$ initio studies [44, 45] we however went back to the original $\kappa$-type lattice structure and treated all molecules as entities. As a consequence, we find evidence for a mixed-symmetry extended $s+d_{x^{2}-y^{2}}$ state, which has been suggested to exist in some parameter regions of various models for $\kappa$-type charge-transfer salts [26, 46, 47, but has so far not been shown to exist in a material-specific Hamiltonian. Interestingly, a different kind of eight node superconductivity has been recently proposed in the context of Fe-based superconductors 48 .

The nodes $\beta$ correspond to the usually discussed $d_{x^{2}-y^{2}}$ solution, while the nodes $\alpha$ lie close to the $k_{x^{-}}$ direction. In contrast to the $d_{x y}$-gap found in Ref. 23 there are no nodes along the $k_{y}$-direction in our results. The twofold rotational symmetry found in our study is very important, because the analysis of many experiments on $\kappa$-(ET) $)_{2} X$ materials is based, possibly mistakenly, on the subtraction of a large twofold symmetric alleged background (see f.i. Refs. 8 and 49) attributed to other effects, such as phonons.

We note that from a study of the suppression of $T_{c}$ with increasing disorder in $\kappa$-NCS, a mixed order parameter has been suggested as an important constraint on models 
of the superconductivity [50]. The anisotropic gap should also appear in related physical properties, such as the electronic part of the specific heat. Calculations of the electronic contribution to the specific heat based on the gap function determined in this work are in favorable agreement with experimental observations, see Ref. 30 .

Summary.- We have studied the organic superconductor $\kappa$ - $(\mathrm{ET})_{2} \mathrm{Cu}\left[\mathrm{N}(\mathrm{CN})_{2}\right] \mathrm{Br}$ in RPA spin-fluctuation theory based on $a b$ initio density functional theory calculations, as well as low-temperature scanning tunneling microscopy and spectroscopy with the tunneling direction parallel to the layered structure. In both theory and experiment we find evidence for three coherence peaks in the spectra of the superconducting state. Our results indicate that the symmetry of the superconducting pairing in $\kappa$-(ET $)_{2} X$ organics might be neither of the $d_{x y}$ and $d_{x^{2}-y^{2}}$ states discussed in previous studies, but of a mixed extended $s+d_{x^{2}-y^{2}}$ type with four nodes located close to the Brillouin zone diagonals and four nodes located on the quasi-1D sheets.

Acknowledgments.- We thank the German Research Foundation (Deutsche Forschungsgemeinschaft, SFB/TR 49) and the Graduate School Materials Science in Mainz for financial support. DG acknowledges helpful discussions with Andreas Kreisel, Peter J. Hirschfeld and Paul C. Canfield. Calculations were performed on the LOEWE-CSC and FUCHS supercomputers of the Center for Scientific Computing (CSC) in Frankfurt am Main, Germany.

* elmers@uni-mainz.de

[1] R. H. McKenzie, Similarities Between Organic and Cuprate Superconductors, Science 278, 5339 (1997).

[2] N. Toyota, M. Lang, and J. Müller, Low-Dimensional Molecular Metals, Springer-Verlag Berlin Heidelberg (2007).

[3] D. J. Van Harlingen, Phase-sensitive tests of the symmetry of the pairing state in the high-temperature superconductors - Evidence for $d_{x^{2}-y^{2}}$ symmetry, Rev. Mod. Phys. 67, 515 (1995).

[4] C. C. Tsuei and J. R. Kirtley, Pairing symmetry in cuprate superconductors, Rev. Mod. Phys. 72, 969 (2000).

[5] M. Lang and J. Müller, in The Physics of Superconductors Vol. II, edited by K. H. Bennemann and J. B. Ketterson, Springer-Verlag Berlin (2004).

[6] K. Kuroki, Pairing Symmetry Competition in Organic Superconductors, J. Phys. Soc. Jpn. 75, 051013 (2006).

[7] B. Powell and R. H. McKenzie, Strong electronic correlations in superconducting organic charge transfer salts, J. Phys.: Condens. Matter 18, R827 (2006).

[8] L. Malone, O. J. Taylor, J. A. Schlueter, and A. Carrington, Location of gap nodes in the organic superconductors $\kappa-(E T)_{2} \mathrm{Cu}(\mathrm{NCS})_{2}$ and $\kappa-(\mathrm{ET})_{2} \mathrm{Cu}\left[\mathrm{N}(\mathrm{CN})_{2}\right] \mathrm{Br}$ determined by magnetocalorimetry, Phys. Rev. B 82, 014522 (2010).
[9] S. Milbradt, A. A. Bardin, C. J. S. Truncik, W. A. Huttema, A. C. Jacko, P. L. Burn, S. C. Lo, B. J. Powell, and D. M. Broun, In-plane superfluid density and microwave conductivity of the organic superconductor $\kappa$ $(\mathrm{ET})_{2} \mathrm{Cu}\left[\mathrm{N}(\mathrm{CN})_{2}\right] \mathrm{Br}$ : Evidence for d-wave pairing and resilient quasiparticles, Phys. Rev. B 88, 064501 (2013).

[10] O. J. Taylor, A. Carrington, and J. A. Schlueter, SpecificHeat Measurements of the Gap Structure of the Organic Superconductors $\kappa-(E T)_{2} \mathrm{Cu}\left[\mathrm{N}(\mathrm{CN})_{2}\right] \mathrm{Br}$ and $\kappa$ $(E T)_{2} \mathrm{Cu}(\mathrm{NCS})_{2}$, Phys. Rev. Lett. 99, 057001 (2007).

[11] O. J. Taylor, A. Carrington, and J. A. Schlueter, Superconductor-insulator phase separation induced by rapid cooling of $\kappa-(E T)_{2} \mathrm{Cu}\left[\mathrm{N}(\mathrm{CN})_{2}\right] \mathrm{Br}$, Phys. Rev. B 77, 060503(R) (2008).

[12] H. Elsinger, J. Wosnitza, S. Wanka, J. Hagel, D. Schweitzer, and W. Strunz, $\kappa-(B E D T$ TTF $)_{2} \mathrm{Cu}\left[\mathrm{N}(\mathrm{CN})_{2}\right] \mathrm{Br}$ : A Fully Gapped Strong-Coupling Superconductor, Phys. Rev. Lett. 84, 6098 (2000).

[13] J. Müller, M. Lang, R. Helfrich, R. Steglich, and T. Sasaki, High-resolution ac-calorimetry studies of the quasi-two-dimensional organic superconductor $\kappa$ (BEDT-TTF $)_{2} \mathrm{Cu}(\mathrm{NCS})_{2}$, Phys. Rev. B 65, 140509(R) (2002).

[14] J. Wosnitza, S. Wanka, J. Hagel, M. Reibelt, D. Schweitzer, and J. A. Schlueter, Thermodynamic properties of quasi-two-dimensional organic superconductors, Synth. Met. 133, 201 (2003).

[15] M. Dion, D. Fournier, M. Poirier, K. D. Truong, and A.M. S. Tremblay, Mixed pairing symmetry in $\kappa$-(BEDTTTF $)_{2} X$ organic superconductors from ultrasonic velocity measurements, Phys. Rev. B 80, 220511(R) (2009).

[16] T. Arai, K. Ichimura, K. Nomura, S. Takasaki, J. Yamada, S. Nakatsuji, and H. Anzai, Tunneling spectroscopy on the organic superconductor $\kappa$-(BEDTTTF $)_{2} \mathrm{Cu}(\mathrm{NCS})_{2}$ using STM, Phys. Rev. B 63, 104518 (2001).

[17] K. Ichimura, M. Takami, and K. Nomura, Direct Observation of $d$-Wave Superconducting Gap in $\kappa-(E T)_{2} \mathrm{Cu}\left[\mathrm{N}(\mathrm{CN})_{2}\right] \mathrm{Br}$ with Scanning Tunneling $\mathrm{Mi}$ croscopy, J. Phys. Soc. Jpn. 77, 114707 (2008).

[18] Y. Oka, H. Nobukane, N. Matsunaga, K. Nomura, K. Katono, K. Ichimura, and A. Kawamoto, Tunneling Spectroscopy in Organic Superconductor $\kappa-(B E D T-T T F-$ $d[3,3])_{2} \mathrm{Cu}\left[\mathrm{N}(\mathrm{CN})_{2}\right] \mathrm{Br}$, J. Phys. Soc. Jpn. 84, 064713 (2015).

[19] S. Diehl, T. Methfessel, U. Tutsch, J. Müller, M. Lang, M. Huth, M. Jourdan, and H. J. Elmers, Disorderinduced gap in the normal density of states of the organic superconductor $\kappa-(B E D T-T T F)_{2} \mathrm{Cu}\left[\mathrm{N}(\mathrm{CN})_{2}\right] \mathrm{Br}, \mathrm{J}$. Phys.: Condens. Matter 27, 265601 (2015).

[20] K. Koepernik and H. Eschrig, Full-potential nonorthogonal local-orbital minimum-basis band-structure scheme, Phys. Rev. B 59, 1743 (1999); http://www.FPLO.de

[21] H. Eschrig and K. Koepernik, Tight-binding models for the iron-based superconductors, Phys. Rev. B 80, 104503 (2009).

[22] D. Guterding, R. Valentí, and H. O. Jeschke, Influence of molecular conformations on the electronic structure of organic charge transfer salts, Phys. Rev. B 92, 081109(R) (2015); In the present work we use the model for $\kappa$-Br in ground state conformation (eclipsed) of the ET molecules and relabelled the crystal axes $(a, b, c)$ to $(b, c, a)$.

[23] J. Schmalian, Pairing due to Spin Fluctuations in Layered Organic Superconductors, Phys. Rev. Lett. 81, 4232 
(1998).

[24] For the difference in hopping structure, see for instance Fig. 1(a) and Fig. 13 in Ref. 25 or Fig. 1 in Ref. 43.

[25] H. Kino and H. Fukuyama, Phase Diagram of TwoDimensional Organic Conductors: (BEDT-TTF $)_{2} X, \mathrm{~J}$. Phys. Soc. Jpn. 65, 2158 (1996).

[26] K. Kuroki, T. Kimura, R. Arita, Y. Tanaka, and Y. Matsuda, $d_{x^{2}-y^{2}}$ versus $d_{x y}$-like pairings in organic superconductors $\kappa-(B E D T-T T F)_{2} X$, Phys. Rev. B 65, 100516(R) (2002).

[27] A. Ardavan, S. Brown, S. Kagoshima, K. Kanoda, K. Kuroki, H. Mori, M. Ogata, S. Uji, and J. Wosnitza, Recent Topics of Organic Superconductors, J. Phys. Soc. Jpn. 81, 011004 (2012).

[28] E. Yusuf, B. J. Powell, and R. H. McKenzie, Antiferromagnetic spin fluctuations in the metallic phase of quasitwo-dimensional organic superconductors, Phys. Rev. B 75, 214515 (2007).

[29] S. Graser, T. A. Maier, P. J. Hirschfeld, and D. J. Scalapino, Near-degeneracy of several pairing channels in multiorbital models for the Fe pnictides, New J. Phys. 11, 025016 (2009).

[30] See Supplemental Material at [URL inserted by publisher], which includes details on experimental crystal orientation, experimental extraction of the superconducting density of states and an analysis of high-resolution specific heat data. We explain the computational formalism including the RPA spin-fluctuation calculations and present a robustness analysis for the fitting procedures.

[31] A. M. Kini, K. D. Carlson, J. D. Dudek, U. Geiser, H. H. Wang, and J. M. Williams, Isotope effect in BEDTTTF based organic superconductors, Synth. Met. 85, 1617 (1997).

[32] L. Pintschovius, H. Rietschel, T. Sasaki, H. Mori, S. Tanaka, N. Toyota, M. Lang, and F. Steglich, Observation of superconductivity-induced phonon frequency changes in the organic superconductor $\kappa-(B E D T$ TTF $)_{2} \mathrm{Cu}(\mathrm{NCS})_{2}$, Europhys. Lett. 37, 627 (1997).

[33] R. C. Dynes, V. Narayanamurti, and J. P. Garno, Direct Measurement of Quasiparticle-Lifetime Broadening in a Strong-Coupled Superconductor, Phys. Rev. Lett. 41, 1509 (1978).

[34] H. Anzai, J. M. Delrieu, S. Takasaki, S. Nakatsuji, and J. Yamada, Crystal growth of organic charge-transfer complexes by electrocrystallization with controlled applied current, J. Cryst. Growth 154, 145 (1995).

[35] J. Müller, M. Lang, F. Steglich, J. A. Schlueter, A. M. Kini, and T. Sasaki, Evidence for structural and electronic instabilities at intermediate temperatures in $\kappa$ $(\mathrm{BEDT}-\mathrm{TTF})_{2} \mathrm{X}$ for $\mathrm{X}=\mathrm{Cu}\left[\mathrm{N}(\mathrm{CN})_{2}\right] \mathrm{Cl}, \mathrm{Cu}\left[\mathrm{N}(\mathrm{CN})_{2} \mathrm{Br}\right.$ and $\mathrm{Cu}(\mathrm{NCS})_{2}$ : Implications for the phase diagram of these quasi-two-dimensional organic superconductors, Phys. Rev. B 65, 144521 (2002).

[36] B. Hartmann, J. Müller, and T. Sasaki, Mott metalinsulator transition induced by utilizing a glasslike structural ordering in low-dimensional molecular conductors, Phys. Rev. B 90, 195150 (2014).

[37] J. Müller, B. Hartmann, R. Rommel, J. Brandenburg, S. M. Winter, and J. A. Schlueter, Origin of the glass-like dynamics in molecular metals $\kappa$-(BEDTTTF $)_{2} X$ : implications from fluctuation spectroscopy and ab initio calculations, New. J. Phys. 17, 083057 (2015).

[38] T. Burgin, T. Miebach, J. C. Huffman, L. K. Montgomery, J. A. Paradis, C. Rovira, M.-H. Whangbo,
S. N. Magonov, S. I. Khan, C. E. Strouse, D. L. Overmyer, and J. E. Schirber, 20 K Crystal Structure, Electrical Transport, Electronic Band Structure, Scanning Tunnelling Microscopy and Pressure-RF Impedance Studies on the Organic Conducting Salt $\kappa$-(BEDT$\mathrm{TSF})_{2} \mathrm{Cu}\left[\mathrm{N}(\mathrm{CN})_{2}\right] \mathrm{Br}$, J. Mater. Chem. 5, 1659 (1995).

[39] V. A. Ukraintsev, Data evaluation technique for electrontunneling spectroscopy, Phys. Rev. B 53, 11176 (1996).

[40] H. Shinaoka and M. Imada, Single-Particle Excitations under Coexisting Electron Correlation and Disorder: A Numerical Study of the Anderson-Hubbard Model, J. Phys. Soc. Jpn. 78, 094708 (2009).

[41] R. H. McKenzie, A strongly correlated electron model for the layered organic superconductors $\kappa$ - $(B E D T-T T F)_{2} X$, Comments Cond. Matt. Phys. 18, 309 (1998).

[42] M. Zehetmayer, A review of two-band superconductivity: materials and effects on the thermodynamic and reversible mixed-state properties, Supercond. Sci. Technol. 26, 043001 (2013).

[43] H. C. Kandpal, I. Opahle, Y.-Z. Zhang, H. O. Jeschke, and R. Valentí, Revision of model parameters for kappatype charge transfer salts: an ab initio study, Phys. Rev. Lett. 103, 067004 (2009).

[44] K. Nakamura, Y. Yoshimoto, and M. Imada, Ab initio two-dimensional multiband low-energy models of $\mathrm{EtMe}_{3} \mathrm{Sb}\left[\mathrm{Pd}(\mathrm{dmit})_{2}\right]_{2}$ and $\kappa$ - $(\mathrm{BEDT}-\mathrm{TTF})_{2} \mathrm{Cu}(\mathrm{NCS})_{2}$ with comparisons to single-band models, Phys. Rev. B 86, 205117 (2012).

[45] M. Altmeyer, R. Valentí, and H. O. Jeschke, Role of layer packing for the electronic properties of the organic superconductor (BEDT-TTF $)_{2} \mathrm{Ag}\left(\mathrm{CF}_{3}\right)_{4}(\mathrm{TCE})$, Phys. Rev. B 91, 245137 (2015).

[46] B. J. Powell and R. H. McKenzie, Symmetry of the Superconducting Order Parameter in Frustrated Systems Determined by the Spatial Anisotropy of Spin Correlations, Phys. Rev. Lett. 98, 027005 (2007).

[47] T. Watanabe, H. Yokoyama, Y. Tanaka, and J. Inoue, Predominant magnetic states in the Hubbard model on anisotropic triangular lattices, Phys. Rev. B 77, 214505 (2008).

[48] K. Okazaki, Y. Ota, Y. Kotani, W. Malaeb, Y. Ishida, T. Shimojima, T. Kiss, S. Watanabe, C-T. Chen, K. Kihou, C-H. Lee, A. Iyo, H. Eisaki, T. Saito, H. Fukazawa, Y. Kohori, K. Hashimoto, T. Shibauchi, Y. Matsuda, H. Ikeda, H. Miyahara, R. Arita, A. Chainani, and S. Shin, Octet-Line Node Structure of Superconducting Order Parameter in $\mathrm{KFe}_{2} \mathrm{As}_{2}$, Science 337, 1314 (2012).

[49] K. Izawa, H. Yamaguchi, T. Sasaki, and Y. Matsuda, Superconducting Gap Structure of $\kappa$-(BEDTTTF $)_{2} \mathrm{Cu}(\mathrm{SCN})_{2}$ probed by Thermal Conductivity Tensor, Phys. Rev. Lett. 88, 027002 (2001).

[50] J. G. Analytis, A. Ardavan, S. J. Blundell, R. L. Owen, E. F. Garman, C. Jeynes, and B. J. Powell, Effect of irradiation-induced disorder on the conductivity and critical temperature of the organic superconductor $\kappa-(B E D T$ TTF $)_{2} \mathrm{Cu}(\mathrm{SCN})_{2}$, Phys. Rev. Lett. 96, 177002 (2006). 


\title{
Evidence for eight node mixed-symmetry superconductivity in a correlated organic metal: Supplemental Information
}

\author{
Daniel Guterding, ${ }^{1}$ Sandra Diehl, ${ }^{2,3}$ Michaela Altmeyer, ${ }^{1}$ Torsten Methfessel, ${ }^{3}$ \\ Ulrich Tutsch, ${ }^{4}$ Harald Schubert, ${ }^{4}$ Michael Lang, ${ }^{4}$ Jens Müller, ${ }^{4}$ Michael Huth, ${ }^{4}$ \\ Harald O. Jeschke, ${ }^{1}$ Roser Valentí, ${ }^{1}$ Martin Jourdan, ${ }^{3}$ and Hans-Joachim Elmers ${ }^{3}$ * \\ ${ }^{1}$ Institut für Theoretische Physik, Goethe-Universität Frankfurt, \\ Max-von-Laue-Str. 1, 60438 Frankfurt am Main, Germany \\ ${ }^{2}$ Graduate School Materials Science in Mainz, Staudingerweg 9, 55128 Mainz, Germany \\ ${ }^{3}$ Institut für Physik, Johannes Gutenberg-Universität Mainz, Staudingerweg 7, 55128 Mainz, Germany \\ ${ }^{4}$ Physikalisches Institut, Goethe-Universität Frankfurt, \\ Max-von-Laue-Str. 1, 60438 Frankfurt am Main, Germany
}

(Dated: May 18, 2016)

\section{EXPERIMENTAL CRYSTAL ORIENTATION}

The height profile measured across the insulating and conducting layers shown in the STM image (Fig. 1 in the main text) carries mostly topographical information. The insulating anion-layers are expected to appear slightly lower (darker) than the conducting BEDT-TTF layers, which is indeed observed but it is, however, a small contribution. Therefore, one can deduce the tilting angle from a comparison to the known crystal structure. By analyzing the height profile along a single unit cell we determine the orientation of the cutting plane (see Fig. 1). Depending on the rotation about the $c$-axis (the axis perpendicular to the conducting layers) the height corrugation caused by the alternating orientation of the BEDT-TTF molecules is more or less pronounced. The best agreement between height profile and crystal structure is found for a tilting angle $\varphi_{a b}=60^{\circ}$ about the $c$-axis with respect to the $b$-axis.

\section{EXPERIMENTAL EXTRACTION OF THE SUPERCONDUCTING DOS}

In STS, the spectra have to be corrected for the different work functions of tip and sample which leads to a voltage-dependent asymmetric tunneling transmission function $T(V)^{1}$. For this reason all spectra shown here are normalized to $T(V)$ which was obtained from spectra taken at $13 \mathrm{~K}$, i.e., in the normal state of $\kappa$-Br. We furthermore assume a constant DOS for the tip material in the relevant energy range so that the $d I / d V / T(V)$ curves reflect the thermally smeared DOS $D(V)$ of the sample with $e V=E-E_{\mathrm{F}}\left(E_{F}\right.$ denotes the Fermi energy). Then we analyze this data in the framework of the AndersonHubbard (AH) model discussed by Shinaoka and Imada ${ }^{2}$ for disordered itinerant electron systems with short-range interactions ${ }^{3}$.

In their numerical treatment of the AH model a scaling law is introduced for the DOS in the presence of shortrange Coulomb interactions and a multi-valley energy landscape $^{2}: \quad B(V)=c \exp \left[-\alpha(-\log |\mathrm{e} V|)^{d}\right],|V| \geq V_{0}$, where $d$ denotes the spatial dimension $(d=2$ for the present case) and $\alpha=0.288$ is a non-universal constant. In the immediate vicinity of $E_{F}$ the $\mathrm{AH}$ model is not applicable ${ }^{2}$. A very good description of this energy region is obtained by assuming a hard energy gap of small size accounted for by a phenomenological DOS function of the following form: $B(V)=c^{\prime} \cosh \left(\frac{\mathrm{e} V}{\varepsilon_{T}}\right),|V|<V_{0}$, where $c^{\prime}$ is a constant and $\varepsilon_{T}$ measures the effective barrier height. For finite temperatures $T>0, B(V)$ rapidly becomes non-zero near $E_{\mathrm{F}}$ because a thermally activated crossing of the small barrier $\varepsilon_{T}$ leads to a nearly temperatureindependent prefactor and the voltage-dependence is described by the cosh term. With the requirement of continuous differentiability at the inflection point $V_{0}, c^{\prime}$ and $\varepsilon_{T}$ terms are fixed for any given temperature and do not represent adjustable parameters. The conductance spectrum of the superconducting state is given by

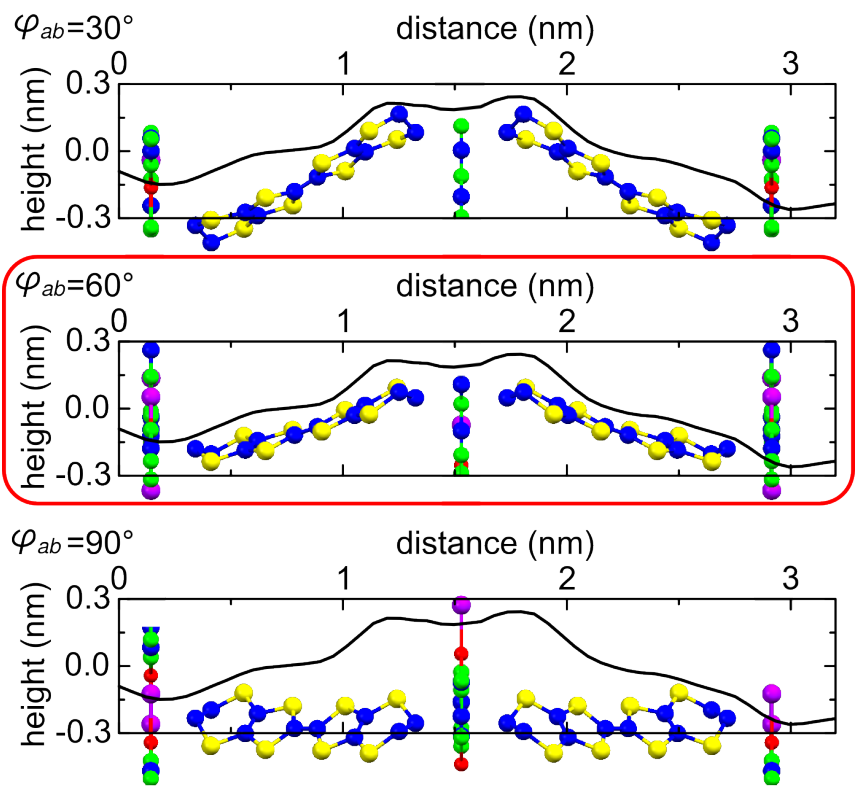

FIG. 1. Height profile along a single unit cell with the corresponding crystal orientation showing the best agreement for an angle for rotation about the $c$-axis of $\varphi_{a b}=60^{\circ}$. 


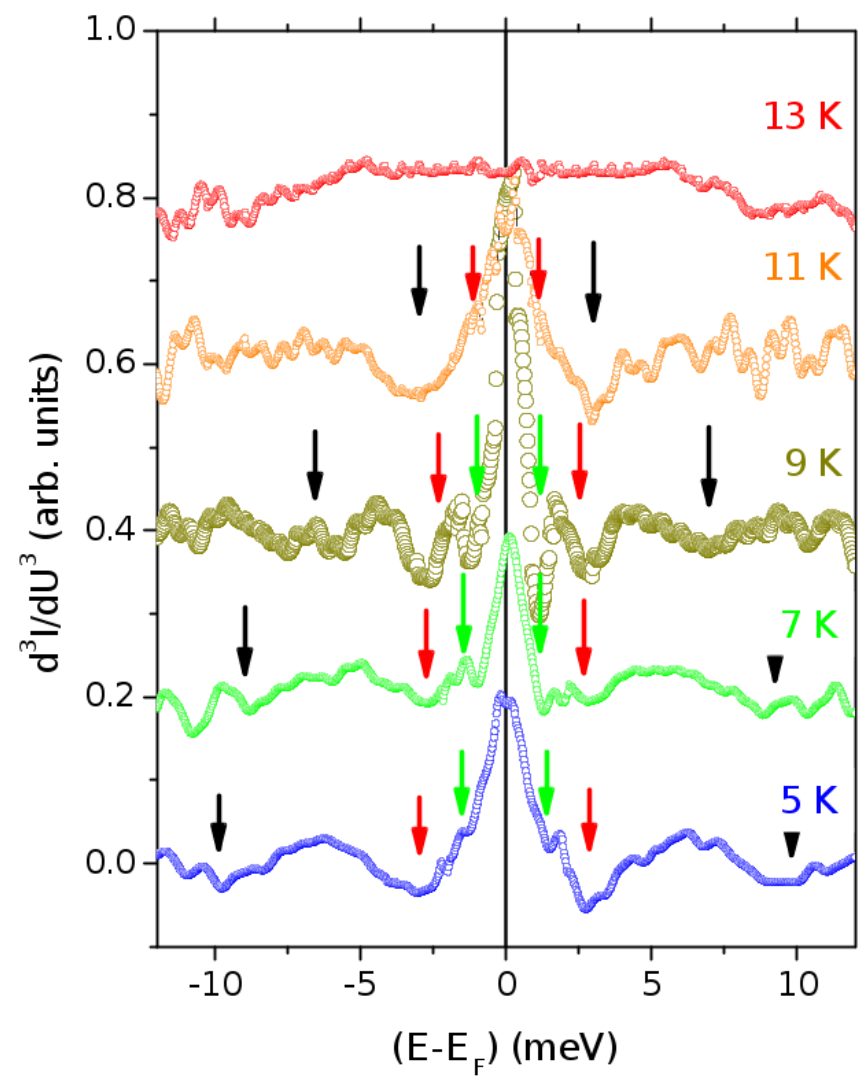

FIG. 2. Temperature dependence of the second derivative of the conductance spectrum $\mathrm{d} I / \mathrm{d} V / T(V)$. Features A, B and $\mathrm{C}$ are indicated by the colored arrows (black, red and green respectively).

$S(V)=d I / d V / T(V) / B(V)$.

\section{TEMPERATURE DEPENDENCE OF FEATURES OBSERVED IN THE RAW CONDUCTANCE}

In Fig. 3 of the main paper we have shown the second derivative of the conductance spectrum $\mathrm{d} I / \mathrm{d} V / T(V)$ at $T=5 \mathrm{~K}$. For completeness, the temperature dependence of this quantity is shown in Fig. 2. The temperature evolution of the superconducting gaps associated with features A, B and C can be traced clearly. All gaps decrease when increasing the temperature towards $T_{c}$. In the spectrum taken at $T=13 \mathrm{~K}>T_{c}$ all signatures of a superconducting gap are gone.

The second derivative of the tunneling conductance was calculated after smoothing the data using the Savitzky-Golay method ${ }^{4}$, which is a widely used technique to increase the signal-to-noise ratio without significantly distorting the signal data. The averaging interval is $2.3 \mathrm{meV}$ in the interval [-2.09:2.09] $\mathrm{meV}$ and $6 \mathrm{meV}$ outside of this interval. Different smoothing intervals are justified because the noise increases with increasing absolute tunneling current. Thus the noise is considerably
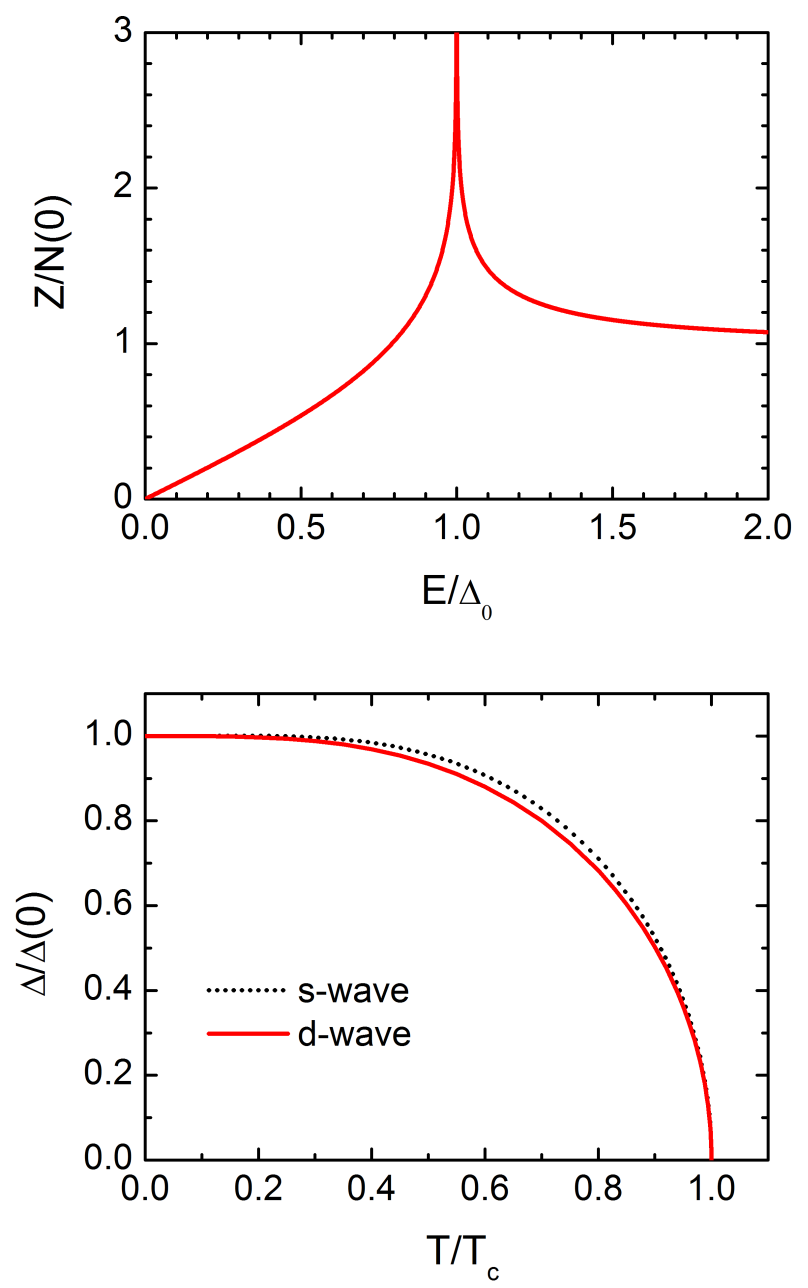

FIG. 3. Density of quasiparticle excitations and energy gap. The upper graph shows the density of quasiparticle excitations in the superconduting state (weak-coupling $d$-wave) normalized to the density of states at the Fermi edge in the normal-conducting state as function of the excitation energy normalized to the maximum value of the energy gap. In the lower graph the normalized energy gap as function of temperature (normalized to $T_{c}$ ) is shown for the weak-coupling $d$-wave (red curve) and $s$-wave (black dotted curve) case.

lower in the close vicinity of $E-E_{F}=0$.

\section{ANALYSIS OF SPECIFIC HEAT MEASUREMENTS}

A method for calculating the specific heat of an unconventional superconductor from first principles has not been developed yet. Therefore, we have performed calculations of the specific heat of $\kappa$ - $\mathrm{Br}$ in the framework of the multi-band alpha model for $d$-wave superconductors in order to check for compatibility of the gap parameters, as derived from our STS data, with specific 
heat results from the literature ${ }^{5}$. The alpha model for $s$ wave superconductors, introduced by Padamsee et al. ${ }^{6}$, extends BCS theory in a purely phenomenological way to materials with arbitrary (positive) values of the ratio $\alpha=\Delta(0) / k_{\mathrm{B}} T_{c}$. As low temperature specific heat data for $\kappa$ - $\mathrm{Br}$ indicate nodes in the gap function ${ }^{5}$ ( $d$-wave) as do our STS results (extended $s+d_{x^{2}-y^{2}}$ ), we extend the alpha model to the $d$-wave case. This can be done once the density of quasiparticle excitations $Z_{d}(E)$ and the gap function $\Delta_{d}(k, T)$ are known for this case. These two quantities have been calculated using BCS theory with a cylindrical Fermi surface and a gap function of the type $\Delta_{d}=\Delta_{0}(T) \cos (2 \varphi)$. As the electronic system of $\kappa$-Br shows a strongly two-dimensional character, these approximations can be considered as an appropriate simplification for estimating the specific heat. The results for $Z_{d}(E)$ and $\Delta_{0}(T)$ are shown in Fig. 3. A value of $\alpha=2.14$ has been obtained for the weak coupling limit consistent with the results of Ref. 7 .

The multi-band alpha model, where each of the gaps occurs in a different band, deviates from the one-band scenario (described in the subsequent theoretical parts) which is used for fitting the STS data. However, as the specific heat depends essentially only on the quasiparticle density of states $Z(E)$, we attempt to fit this quantity (known from the theoretical considerations and the fitting of the STS data) by a sum of $d$-wave quasiparticle densities of states, each with its own maximum gap value $\Delta_{0, i}(T)$, i.e., with a multi-band alpha model. As an approximation to the density of states derived by the microscopic theory (blue line in Fig. 4) we use the function

$$
[Z(E) / N(0)]_{\mathrm{fit}}=\sum_{i=1}^{n} g_{i} z_{d}\left(E / \Delta_{0, i}(T)\right)
$$

with $z_{d}\left(E / \Delta_{0, i}(T)\right)$ and $\Delta_{0, i}(T) / \Delta_{0, i}(0)$ being the $d$ wave functions shown in Fig. $3, N(0)$ the density of states at the Fermi edge in the normal-conducting state and $\sum_{i=1}^{n} g_{i}=1$. Here $g_{i}$ and $\Delta_{0, i}(0)$ are used as adjustable parameters. In our case three bands are needed, as the quasiparticle density of states shows three local maxima (see Fig. 4). The so-derived fitting function $[Z(E) / N(0)]_{\text {fit }}$ with the parameter values $g_{1}=0.08$, $g_{2}=0.54, g_{3}=0.38$ and $\Delta_{0,1}(0)=0.48 \mathrm{meV}, \Delta_{0,2}(0)=$ $2.58 \mathrm{meV}, \Delta_{0,3}(0)=9.20 \mathrm{meV}$ is displayed in Fig. 4 (red dotted curve) and shows a fairly good agreement with the theoretical results. Using a $T_{c}$ value of $11.2 \mathrm{~K}$, as obtained from the theoretical fit to the STS derived $\Delta_{0}(T)$ points, we get $\alpha_{1}=0.50, \alpha_{2}=2.67, \alpha_{1}=9.53$. The specific heat of the three-band alpha model is then given by

$$
C=\sum_{i=1}^{3} C_{d}\left(T, T_{c}, \gamma_{i}, \alpha_{i}\right)
$$

with $\gamma_{i}=g_{i} \gamma$. The result of this calculation for the specific heat is shown in Fig. 5 (blue curve) together with

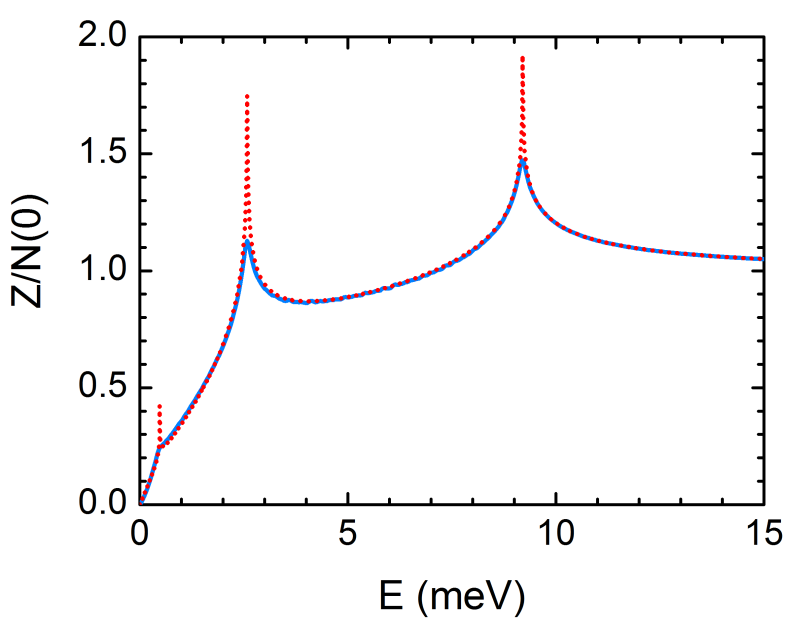

FIG. 4. Density of quasiparticle excitations for $\kappa$-Br. Normalized quasiparticle density of states for the parameter values (at $T=5 \mathrm{~K}$ ) given in Table I of the main text with a small broadening of $\Gamma=0.07 \mathrm{meV}$ (blue curve) and for the threeband alpha model (red dotted curve) with $g_{1}=0.08, g_{2}=$ $0.54, g_{3}=0.38$ and $\Delta_{0,1}(0)=0.48 \mathrm{meV}, \Delta_{0,2}(0)=2.58 \mathrm{meV}$, $\Delta_{0,3}(0)=9.20 \mathrm{meV}$.

the literature data from Ref. 5. The $\gamma$ value was set to $27.5 \mathrm{~mJ} /\left(\mathrm{mol} \mathrm{K}^{2}\right)$ as determined by Ref. 5 and a Gaussian $T_{c}$ distribution $\left(\sigma=0.98 \mathrm{~K}, \bar{T}_{c}=12.0 \mathrm{~K}\right)$ has been used to take into account the rounded maximum of the experimental data. The calculated results are in fairly good agreement with the experimental data. A better (and nearly perfect) fit can be obtained (red curve) by changing the $g_{i}$ values to $g_{1}=0.04, g_{2}=0.76, g_{3}=0.20$ and setting $\alpha_{2}=3.10$ (accounting for the dominant contribution to the specific heat), $\sigma=0.83 \mathrm{~K}, \bar{T}_{c}=12.1 \mathrm{~K}$.

\section{THEORY: BCS DENSITY OF STATES}

The basic idea of BCS theory is the Cooper problem, which states that an attractive interaction between two electrons with opposite spin and momentum leads to a bound state, no matter how small it is. The corresponding Hamiltonian for Cooper pairs having a net momentum of zero can be written as

$$
H=\sum_{k, \sigma} \epsilon_{k \sigma} c_{k \sigma}^{\dagger} c_{k \sigma}+\sum_{k, k^{\prime}} U\left(k, k^{\prime}\right) c_{k \uparrow}^{\dagger} c_{-k \downarrow}^{\dagger} c_{-k^{\prime} \downarrow} c_{k^{\prime} \uparrow} .
$$

The interaction can be treated in mean field theory $\left(\delta\left(c^{\dagger} c^{\dagger}\right)=c^{\dagger} c^{\dagger}-\left\langle c^{\dagger} c^{\dagger}\right\rangle\right)$, where terms quadratic in $\delta$ are neglected. The resulting Hamiltonian can be diagonalized using the Bogoliubov-Valatin transformation, which introduces quasiparticle creation and annihilation operators $\gamma_{k \sigma}^{\dagger}$ and $\gamma_{k \sigma}$. The quasiparticle excitation energies are given as $E_{k}=\sqrt{\epsilon_{k}^{2}+\left|\Delta_{k}\right|^{2}}$, where $\Delta(k)=$ $\sum_{k^{\prime}} U\left(k, k^{\prime}\right)\left\langle c_{-k^{\prime} \downarrow} c_{k^{\prime} \uparrow}\right\rangle$. 


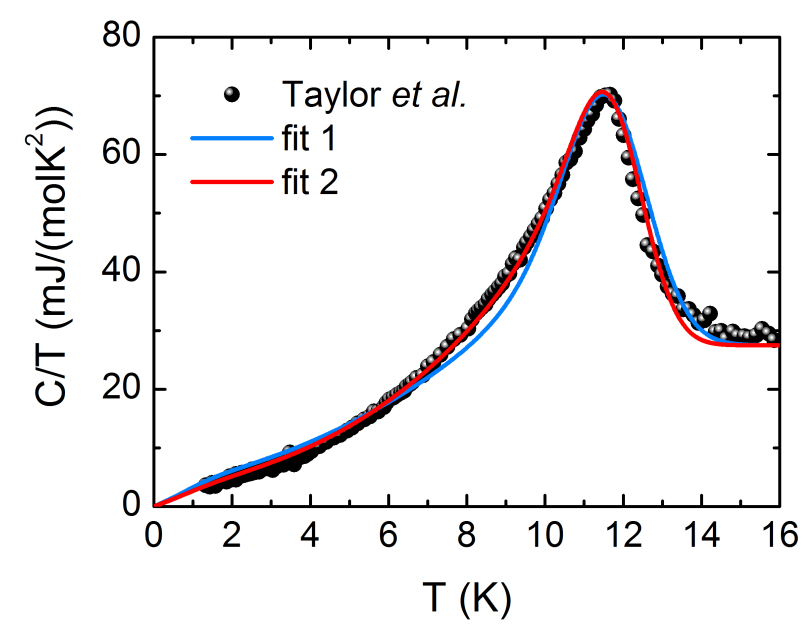

FIG. 5. Specific heat (devided by temperature). Black spheres are data from Taylor et al. ${ }^{5}$, blue and red lines indicate our fits with the three-band alpha model for $d$-wave superconductors and a gaussian $T_{c}$ distribution. The parameters of fit 1 (fit 2) are: $\gamma=27.5 \mathrm{~mJ} /\left(\mathrm{mol} \mathrm{K}^{2}\right), g_{1}=0.08(0.04)$, $g_{2}=0.54(0.76), g_{3}=0.38(0.20), \alpha_{1}=0.50, \alpha_{2}=2.67(3.10)$, $\alpha_{3}=9.65$ and $\bar{T}_{c}=12.0 \mathrm{~K}(12.1 \mathrm{~K}), \sigma=0.98 \mathrm{~K}(0.83 \mathrm{~K})$.

The BCS Hamiltonian in terms of the quasiparticle creation and annihilation operators reads

$$
\begin{aligned}
H_{\mathrm{BCS}}= & \sum_{k, \sigma} E_{k} \gamma_{k \sigma}^{\dagger} \gamma_{k \sigma}+\sum_{k} \epsilon_{k} \\
& -\sum_{k, k^{\prime}} U\left(k, k^{\prime}\right)\left\langle c_{k \uparrow}^{\dagger} c_{-k \downarrow}^{\dagger}\right\rangle\left\langle c_{-k^{\prime} \downarrow} c_{k^{\prime} \uparrow}\right\rangle .
\end{aligned}
$$

The excitation spectrum of the quasiparticles $E_{k}$ is gapped and defined only for positive energies. The density of states of quasiparticles in an isotropic s-wave superconductor $\left(\Delta_{k}=\Delta_{0}\right)$ can be calculated via

$$
\begin{aligned}
\rho_{\mathrm{qp}}(E) & =\frac{1}{N} \sum_{k} \delta\left(E-E_{k}\right) \\
& =\int d \epsilon \rho_{0}(\epsilon) \frac{\sqrt{\epsilon^{2}+|\Delta|^{2}}}{\epsilon} \delta\left(\epsilon-\sqrt{E^{2}-|\Delta|^{2}}\right) \\
& = \begin{cases}\rho_{0}\left(\sqrt{E^{2}-|\Delta|^{2}}\right) \frac{E}{\sqrt{E^{2}-|\Delta|^{2}}} & E>|\Delta| \\
0 & E<|\Delta|\end{cases}
\end{aligned}
$$

As the quasiparticles are superpositions of particles and holes, this is not the density of states that can be measured in, for instance, scanning tunneling spectroscopy. To determine the electron DOS, we have to start from another commonly used expression of the density of states in terms of Greens functions, $\rho(E)=$ $-\frac{1}{\pi} \sum_{k \sigma} \operatorname{Im}\left(-\left\langle c_{k \sigma}^{\dagger} c_{k \sigma}\right\rangle_{0}\right)$.

We assume a non spin-polarized energy dispersion and

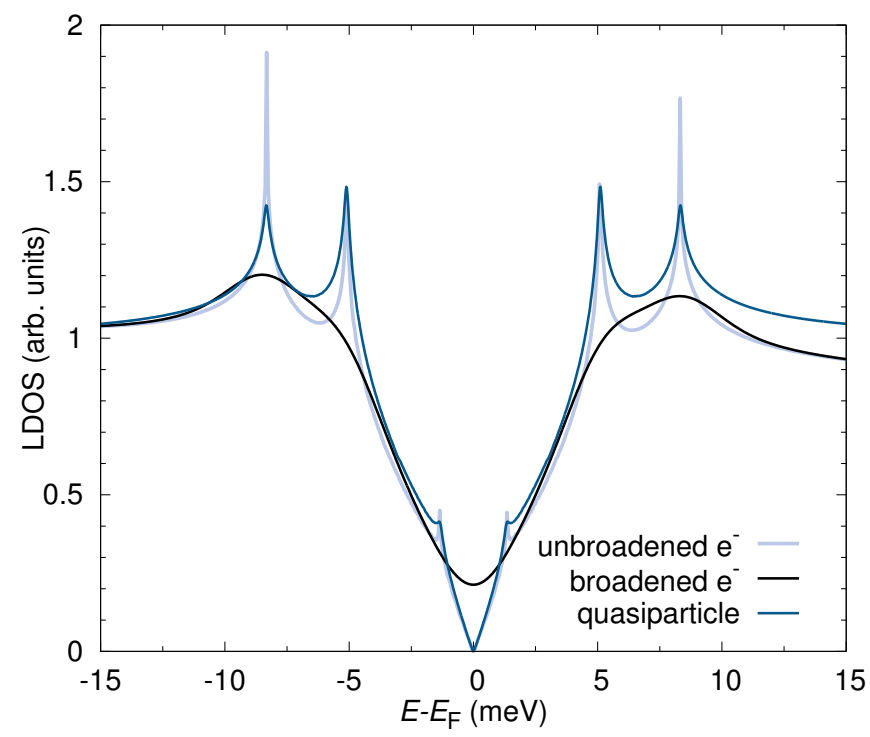

FIG. 6. Comparison of broadened and unbroadened electron DOS to the quasiparticle DOS for identical parameters (as given in Table I). The quasiparticle DOS includes a small broadening of $\Gamma=0.07 \mathrm{meV}$. The energy scale is set to $\Delta_{0}=10 \mathrm{meV}$.

again insert the Bogoliubov-Valatin transformation

$$
\rho(E)=\frac{2}{\pi} \sum_{k} \operatorname{Im}\left(\left|u_{k}\right|^{2}\left\langle\gamma_{k \uparrow}^{\dagger} \gamma_{k \uparrow}\right\rangle_{0}+\left|v_{k}\right|^{2}\left\langle\gamma_{-k \downarrow} \gamma_{-k \downarrow}^{\dagger}\right\rangle_{0}\right) .
$$

As the Hamiltonian is diagonal in the quasiparticle operators, we can insert the expression for the bare Greens function and get

$$
\rho(E)=2 \sum_{k}\left(\left|u_{k}\right|^{2} \delta\left(E-E_{k}\right)+\left|v_{k}\right|^{2} \delta\left(E+E_{k}\right)\right),
$$

where $k$ is a combined momentum and band index. $\left|u_{k}\right|^{2}$ and $\left|v_{k}\right|^{2}$ are the probabilities for the excitations being hole- or electron-like respectively. Only positive energies are taken into account ${ }^{8}$.

Next we calculate the DOS explicitly for the ab initio derived bandstructure and the superconducting gap calculated microscopically. The delta functions in the DOS are replaced by gaussians with a standard deviation of $1.2 \mathrm{meV}$ to simulate the broadening observed in experiment. For comparison we also calculated the DOS with a tetrahedron method ${ }^{9}$ that does not employ any broadening. For the calculation of the DOS using gaussians we included $4000 \times 4000 \mathrm{k}$-points in the xy-plane. In the tetrahedron method we used $4000 \times 4000 \times 2$ k-points.

A comparison of the broadened and unbroadened electron DOS and the quasiparticle DOS is shown in Fig. 6 . Peak positions are identical. Only the background far away from the Fermi level is not well represented by the quasiparticle approximation. This can be easily understood from the structure of the expression derived before. To one energy $E$ two different electron energies $\epsilon_{k}$ and 


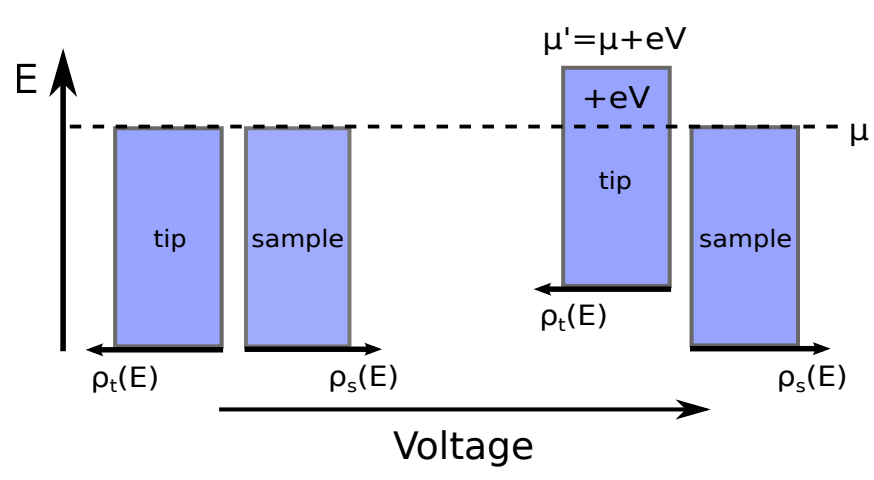

FIG. 7. Illustration of the energy levels in tip and sample with (right) and without (left) applied voltage. The voltage raises the energy levels of the tip, so that the chemical potential is higher than in the sample and a net current results. Note, that these energy shifts in the tip can be described in two ways: $f\left(E, \mu^{\prime}=\mu+e V\right)$ or $f(E-e V, \mu)$.

$\epsilon_{k^{\prime}}$ (where $\epsilon_{k}=-\epsilon_{k^{\prime}}$ ) contribute and the BV coefficients should average to approximately $\sqrt{0.5}$. The small deviations are causing the asymmetric behavior, which merges to the normal density of states far away from the gap.

In the case of an anisotropic gap, the normal state density of states can not be identified easily, because the gap $\Delta$ then also depends on momentum $k$ and the Fermi surface is not a concentric circle.

$$
\begin{aligned}
\rho_{s}(E) & =\int d \epsilon \frac{1}{N} \sum_{k} \delta\left(\epsilon-\epsilon_{k}\right) \delta\left(|E|-\sqrt{\epsilon^{2}+\left|\Delta_{k}\right|^{2}}\right) \\
& \neq \int d \epsilon \rho_{N}(\epsilon) \delta\left(|E|-\sqrt{\epsilon^{2}+\left|\Delta_{k}\right|^{2}}\right) .
\end{aligned}
$$

However, in the commonly used ansatz the electrons are considered to be free (Fermi surface is a concentric circle) and the gap is only determined by the angle $\theta$

$$
\rho_{s}(E)=\frac{1}{(2 \pi)^{2}} m_{e} \operatorname{Re} \int d \theta \frac{|E|}{\sqrt{E^{2}-|\Delta(\theta)|^{2}}} .
$$

For all further calculations we will use this expression for the quasiparticle DOS.

\section{THEORY: DIFFERENTIAL CONDUCTANCE}

In a scanning tunneling spectroscopy (STS) experiment the current from the tip to the sample should obey (see Fig. 7),

$$
\begin{aligned}
I_{t s}(V)= & \frac{2 \pi e}{\hbar} \int_{-\infty}^{\infty} d E\left|M_{t s}(E-e V)\right|^{2} \rho_{\text {tip }}(E-e V) \\
& \times f(E-e V) \rho_{\text {sample }}(E)(1-f(E)),
\end{aligned}
$$

where $e$ is the positive elementary charge and a positive voltage $V$ corresponds to net charge carrier transport into the sample. The current is proportional to the tunneling probability $\left|M_{t s}(E)\right|^{2}$, which depends on tip and sample geometry. Furthermore, the tunneling probability is proportional to the number of occupied states in the tip $\rho_{\text {tip }}(E-e V) f(E-e V)$ and the number of unoccupied states in the sample at lower energies $\rho_{\text {sample }}(E)(1-f(E))$.

After shifting the integration variable $E \rightarrow E+e V$, we arrive at the literature form of the expression for the tunneling current. In order to obtain the net current, we have to calculate the difference between the currents from tip to sample and sample to tip. We obtain

$$
\begin{aligned}
I(V)= & \frac{2 \pi e}{\hbar} \int_{-\infty}^{\infty} d E\left|M_{t s}(E)\right|^{2} \rho_{\text {tip }}(E) \rho_{\text {sample }}(E+e V) \\
& \times(f(E)-f(E+e V)) .
\end{aligned}
$$

The tunneling matrix element $\left|M_{t s}(E)\right|^{2}$ is experimentally not accessible and therefore the tunneling current is divided by the voltage dependent tunneling transmission function $T(V)$, which can be determined from the experimental setup. The density of states of the tip is considered to be independent of energy in the region of interest. We obtain the differential conductance (shifting once again the integration variable $E \rightarrow E-e V$ ) by taking the derivative with respect to the voltage $V$,

$$
\begin{aligned}
& \frac{1}{T(V)} \frac{d I(V)}{d V} \\
= & \frac{2 \pi e}{\hbar} \rho_{\mathrm{tip}}\left(E_{F}\right) \times \int_{-\infty}^{\infty} d E \rho_{\text {sample }}(E)\left(-\frac{d f(E+e V)}{d V}\right) .
\end{aligned}
$$

\section{THEORY: BROADENING}

In the preceding expression we already included thermal broadening via the Fermi function. Additionally we have to take into account the finite quasiparticle lifetime. This can be done (following Dynes et al. ${ }^{10}$ ) by replacing the energies $E$ by $E+i \Gamma$ in the density of states. If we now consider only proportionalities, we can also insert the angle dependent expression for the sample density of states

$$
\begin{aligned}
\frac{d I(V)}{d V} \propto & T(V) \int_{-\infty}^{\infty} d E \int_{0}^{2 \pi} d \theta \\
& \times \operatorname{Re} \frac{|E+i \Gamma|}{\sqrt{(E+i \Gamma)^{2}-\Delta(\theta)^{2}}}\left(-\frac{d f(E+e V)}{d V}\right) .
\end{aligned}
$$

Note that this formula can not be used to fit data with large finite values of the differential conductance at zero bias voltage, since the superconducting DOS goes to zero for zero bias voltage, independent of the choice of $\Gamma$.

Sometimes a slightly different expression is used for the Dynes broadening: $\left|\operatorname{Re}\left[(E+i \Gamma) / \sqrt{(E+i \Gamma)^{2}-\Delta(\theta)^{2}}\right]\right|$ Although only taking the real part and calculating the absolute value were exchanged here, this formula yields a finite value for the superconducting DOS at zero bias voltage, which is proportional to $\Gamma$. Therefore, one can 


\begin{tabular}{rrrr}
\hline \hline name & function & coefficient & value \\
\hline$s \pm$ & $\cos k_{x}+\cos k_{y}$ & $c_{s_{1}}$ & 0.069 \\
$s \pm$ & $\cos k_{x} \cdot \cos k_{y}$ & $c_{s_{2}}$ & -0.672 \\
$d_{x^{2}-y^{2}}$ & $\cos k_{x}-\cos k_{y}$ & $c_{d_{1}}$ & -0.259 \\
$d_{x y}$ & $\sin k_{x} \cdot \sin k_{y}$ & $c_{d_{2}}$ & 0.000 \\
\hline \hline
\end{tabular}

TABLE I. Basis functions considered in the fitting process of the microscopic result for the gap function. Given values are the result of the fitting procedure.

use it to fit data for the differential conductance, which include a constant background. Unfortunately, exchanging the order of the real part and absolute value operations is inconsistent with the expression for the BCS density of states. We fix this problem by introducing the shift of the background explicitly.

\section{THEORY: FITTING GAP SYMMETRIES TO STS DATA}

On top of the broadening effects, it was found that the measured density of states is suppressed by the formation of a soft Hubbard gap caused by short-range interactions and disorder ${ }^{2}$. This correction can be taken into account by scaling the density of states by $B(V)$ as described before. Including this correction the differencial conductance reads

$$
\begin{aligned}
\frac{1}{B(V) T(V)} \frac{d I(V)}{d V} \propto & \int_{-\infty}^{\infty} d E \int_{0}^{2 \pi} d \theta \\
& \times \operatorname{Re} \frac{|E+i \Gamma|}{\sqrt{(E+i \Gamma)^{2}-\Delta(\theta)^{2}}} \\
& \times\left(-\frac{d f(E+e V)}{d V}\right) .
\end{aligned}
$$

For anisotropic gaps on Fermi surfaces, which are not concentric circles, this equation is still an approximation. To improve our calculations, we execute the integration over the angle as a summation over points on the discretized two-dimensional ab initio Fermi surface. We find from our microscopic calculations that the symmetry of the superconducting gap can be described by a superposition of two $s \pm$ and the $d_{x^{2}-y^{2}}$ functions, $\Delta\left(k_{x}, k_{y}\right)=\Delta_{0}\left[\left(c_{s_{1}}\left(\cos \left(k_{x}\right)+\cos \left(k_{y}\right)\right)+\right.\right.$ $\left.c_{s_{2}} \cos \left(k_{x}\right) \cos \left(k_{y}\right)+c_{d_{1}}\left(\cos \left(k_{x}\right)-\cos \left(k_{y}\right)\right)\right]$, where the normalized prefactors $c_{i}\left(\sum_{i}\left|c_{i}\right|=1\right)$ can be a) fitted to the experiment (see Fig. 8), or b) set to the microscopic values (see Fig. 9), so that only one common prefactor $\Delta_{0}$ is fitted to the magnitude of the gap.

The Dynes broadening $\Gamma$ is used as a fit parameter. The value for the differential conductance in the measured data after dividing by correction terms $B(V)$ and $T(V)$ is still finite. This finite differential conductance at zero bias voltage is too large to be explained by thermal broadening. Therefore, we introduce a parameter $x$, which scales and shifts the calculated DOS so that a constant background in the differential conductance is subtracted, while keeping the data points far away from the Fermi level at unity. With this additional correction we obtain the final formula given in the main text.

The gap on the Fermi surface is shown in Fig. 10 for both the microscopic ratios and those obtained from the fit to the STS data. The analytic representation for the microscopic results was determined by fitting a linear combination of symmetry functions given in Table I. The largest deviation of the fit from the microscopic gap per evaluated k-point is $4.55 \%$ of the maximum gap value. The average deviation of the fit is $1.25 \%$ of the maximum gap value. For a comparison of the microscopic result to its analytic representation see Fig. 10.

While Fig. 10 emphasizes the qualitative agreement between experiment and theory, we include also polar and linear plots (see Fig. 11) of the same data to reveal some quantitative differences. The angle $\varphi$ in the $k_{x}-k_{y}$ plane is measured from the $k_{x}$ direction. For the RPA curves and the fitted result the energy scale was set to $\Delta_{0}=10 \mathrm{meV}$ and $\Delta_{0}=12.1 \mathrm{meV}$ respectively. In this way, the RPA result can be compared directly to the data shown in Fig. 6 or the DOS shown in Fig. 4 of the main paper, while the data obtained by analysing the experiment can be compared to Fig. 3 in the main paper.

While the symmetry of the superconducting gap obtained from theory is identical to the one found by fitting the experiment, the size of the superconducting gap around $\varphi \approx 45^{\circ}$ is clearly overestimated in our calculation. Quantitative differences are however to be expected, as our RPA approach does not take into account the electronic self-energy and lacks a selfconsistency condition. Note that there is currently no state-of-the-art method that can quantitatively predict the momentum-resolved gap structure or superconducting transition temperature of two-dimensional correlated electron systems.

Finally, we investigate whether a nodeless anisotropic s-wave symmetry could be present in the system. We combine a plain s-wave gap with the $\mathrm{d}$-wave expressions given in our manuscript and determine the prefactors for the contributions that lead to optimal agreement with the experimental spectrum measured at $5 \mathrm{~K}$ (see Fig. 12). For both the $s+d_{x y}$ and $s+d_{x^{2}-y^{2}}$ case we observe that the $d$-wave component is dominant in the optimal fit to the STS data. This excludes a nodeless anisotropic swave symmetry. The agreement of the $s+d_{x y}$ symmetry is considerably worse than for the $s+d_{x^{2}-y^{2}}$ symmetry. The $s+d_{x^{2}-y^{2}}$ and the $s_{ \pm}+d_{x^{2}-y^{2}}$ solution presented in our manuscript describe the data equally well. However, as a plain s-wave component cannot be caused by antiferromagnetic spin-fluctuations alone, we decided to use the physically more meaningful representation in terms of an extended s-wave component. 

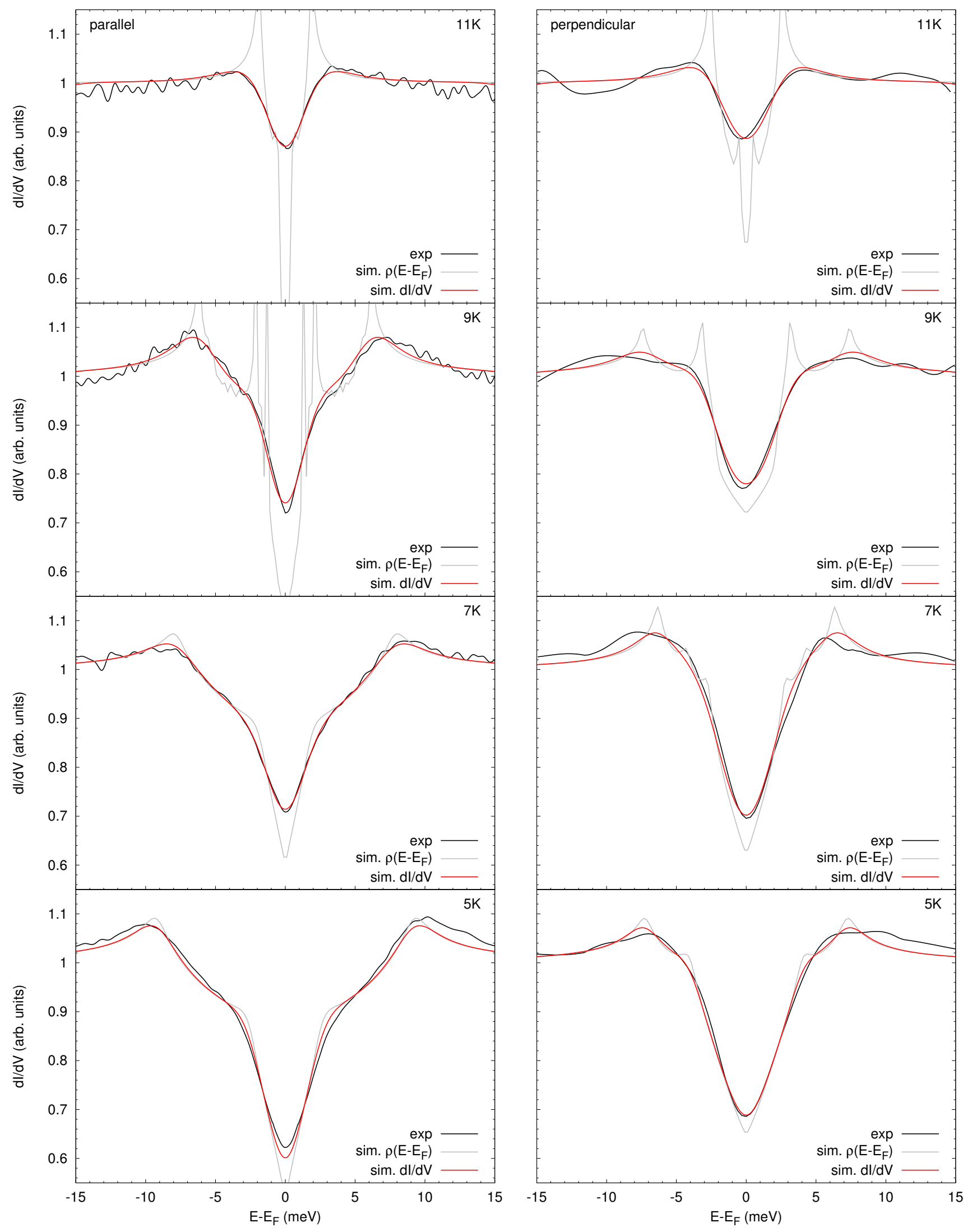

FIG. 8. Fit to the experimental data at the different temperatures (Prefactors are fitted). 

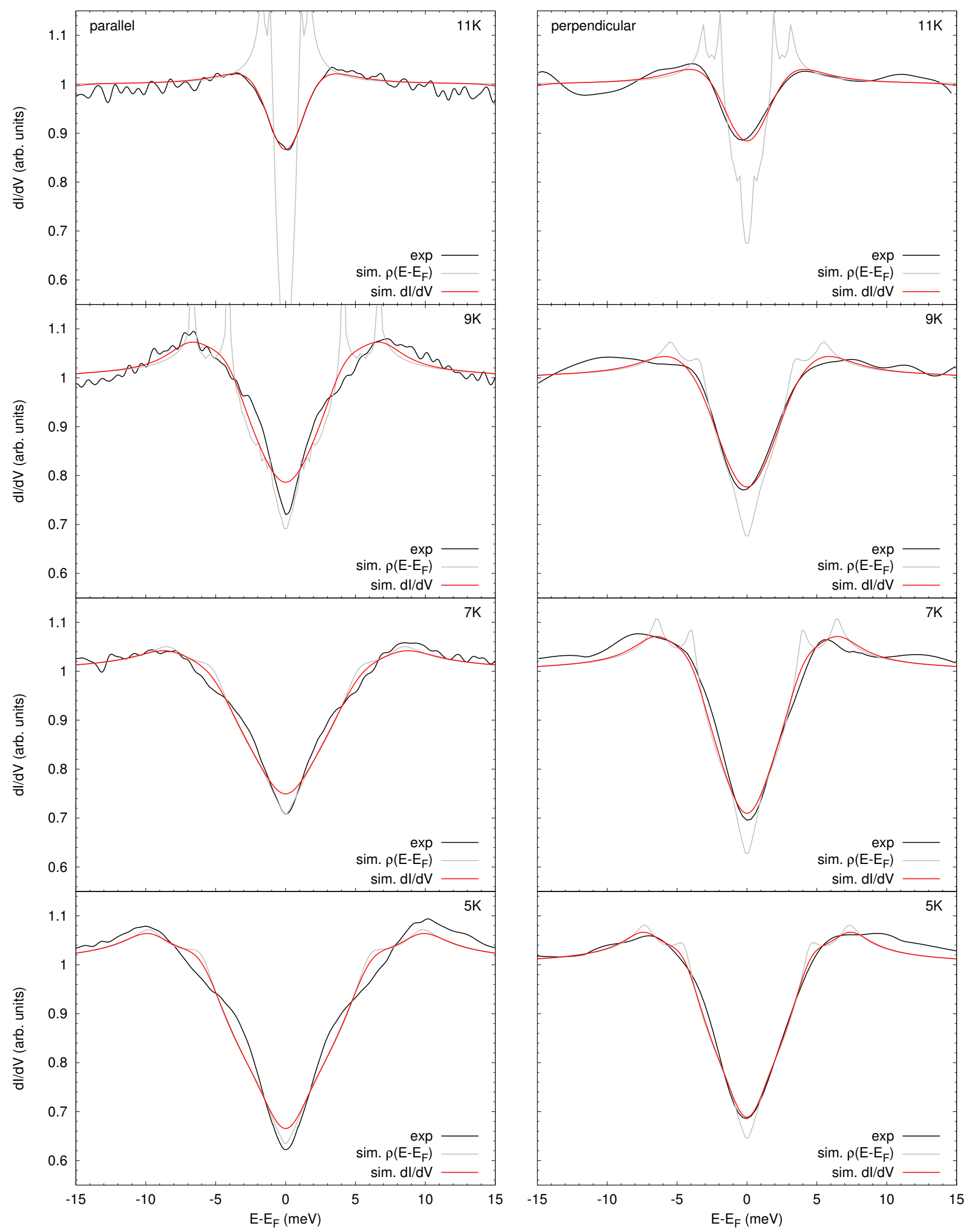

FIG. 9. Fit to the experimental data at the different temperatures (Prefactors fixed to microscopic values). 

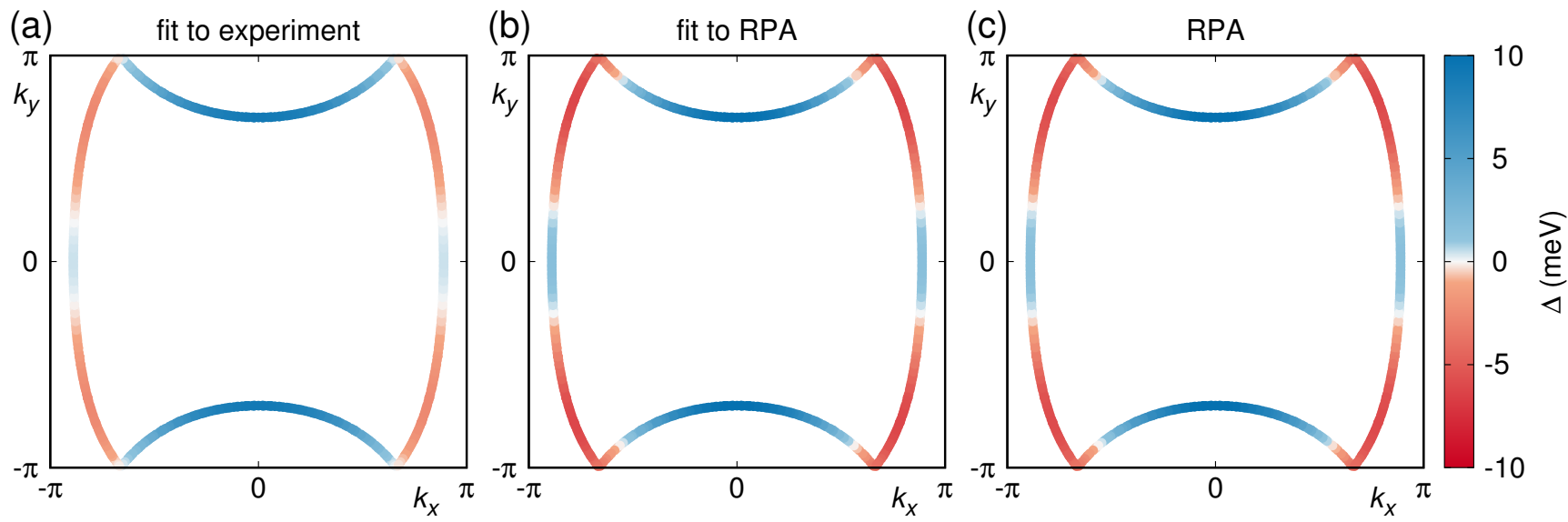

FIG. 10. Comparison of the gap functions on the ab initio Fermi surface obtained from (a) fitting the STS experiment (b) fitting the microscopic result calculated using RPA and (c) the microscopic result itself. All data points of the symmetry functions were multiplied by the energy scale $\Delta_{0}=12.1 \mathrm{eV}$.

\section{THEORY: TEMPERATURE DEPENDENCE OF THE GAP}

To estimate values for the critical temperature and the maximum gap size at zero temperature, we fitted our temperature dependent gap values to the interpolation formula $\Delta(T)=\Delta_{0} \tanh \left(1.74 \sqrt{\frac{T_{c}}{T}-1}\right)$ for the solution of the s-wave BCS self consistency equation (see Fig. 13).

Solving the BCS self-consistency equation for unconventional pairing mechanisms is difficult due to the momentum dependence of the pairing interaction. Therefore, we decided to use the s-wave solution as a rough approximation.

Our predicted values for the critical temperatures $T_{c}=$ $10.3 \pm 1.2 \mathrm{~K}$ for fitted prefactors and $T_{c}=11.2 \pm 0.2$ $\mathrm{K}$ for fixed prefactors are in good agreement with the experimental observation of $T_{c} \approx 11.5 \mathrm{~K}$ (see f.i. Ref. 11). The maximal gap size is found to be $\Delta_{0}=12.9 \pm 2.0$ $\mathrm{meV}$ (fitted prefactors) and $\Delta_{0}=12.1 \pm 0.7 \mathrm{meV}$ (fixed prefactors).

\section{THEORY: AB-INITIO CALCULATIONS}

We use the experimental crystal structure ${ }^{12}$, but relax the ethylene endgroups of the BEDT-TTF molecules in eclipsed configuration ${ }^{13}$. For the exchange correlation functional we use the generalized gradient approximation $(\mathrm{GGA})^{14}$. The DFT calculation was converged using $6 \times$ $6 \times 6$ k-point grids.

\section{THEORY: RPA SPIN-FLUCTUATION PAIRING, FORMALISM}

In $\kappa$-(BEDT-TTF $)_{2} \mathrm{X}$ materials there is strong evidence for antiferromagnetic spin-fluctuations ${ }^{15}$. There- fore, we investigate the superconducting state of these materials based on a random phase approximation (RPA) spin-fluctuation approach ${ }^{16}$. We have generalized our implementation from single-site multi-orbital models ${ }^{17,18}$ to multi-site single-orbital models relevant for the materials discussed here.

The low-energy Hamiltonian is given by the kinetic part $H_{0}$, derived with the Wannier function method described in the main text, and the intra-orbital Hubbard interaction $H_{\mathrm{int}}$.

$$
\begin{aligned}
H & =H_{0}+H_{\mathrm{int}} \\
& =\sum_{\sigma} \sum_{<i j>} t_{i j} c_{i \sigma}^{\dagger} c_{j \sigma}+\frac{U}{2} \sum_{\sigma} \sum_{i} n_{i \sigma} n_{i \bar{\sigma}}
\end{aligned}
$$

Here, $\sigma$ represents the spin and $n_{i \sigma}=c_{i \sigma}^{\dagger} c_{i \sigma}$. The sum over $i$ runs over all BEDT-TTF sites in the unit cell. The hopping integrals $t_{i j}$ taken into account are not restricted to nearest or next-nearest neighbor hoppings. We rather determine the optimal distance cutoff during the wannierization procedure. The interaction strength $U$ is treated as a parameter.

We calculate the non-interacting static susceptibility $\chi^{0}$, where matrix elements $a_{\mu}^{l}(\vec{k})$ resulting from the diagonalization of the initial Hamiltonian $H_{0}$ connect orbital and band-space denoted by indices $l$ and $\mu$ respectively. The $E_{\mu}$ are the eigenvalues of $H_{0}$ and $f(E)$ is the Fermi function.

$$
\begin{aligned}
\chi_{s p q t}^{0}(\vec{q})=-\frac{1}{N_{k}} \sum_{\vec{k}, \mu, \nu} & a_{\mu}^{s}(\vec{k}) a_{\mu}^{p *}(\vec{k}) a_{\nu}^{q}(\vec{k}+\vec{q}) a_{\nu}^{t *}(\vec{k}+\vec{q}) \\
& \times \frac{f\left(E_{\nu}(\vec{k}+\vec{q})\right)-f\left(E_{\mu}(\vec{k})\right)}{E_{\nu}(\vec{k}+\vec{q})-E_{\mu}(\vec{k})}
\end{aligned}
$$

In our calculation both $\vec{q}$ and $\vec{k}$ run over uniform grids spanning the reciprocal unit cell. Temperature enters the calculation through the Fermi functions. 
(a)

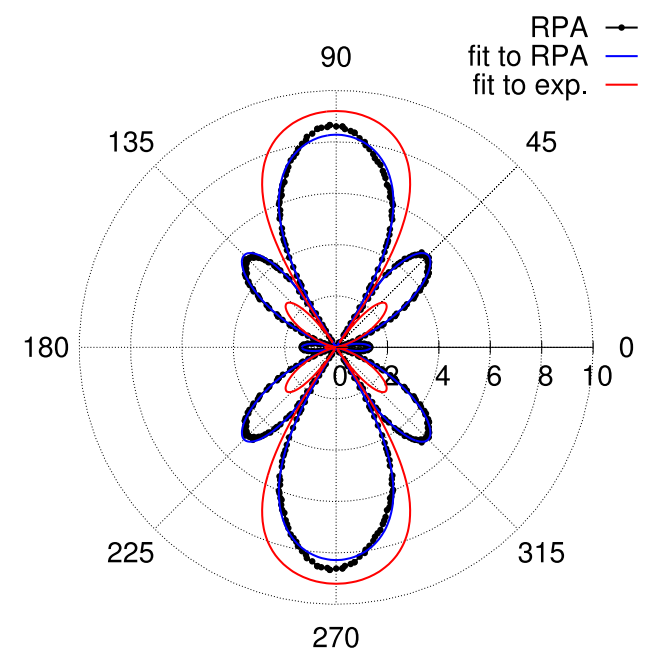

(b)

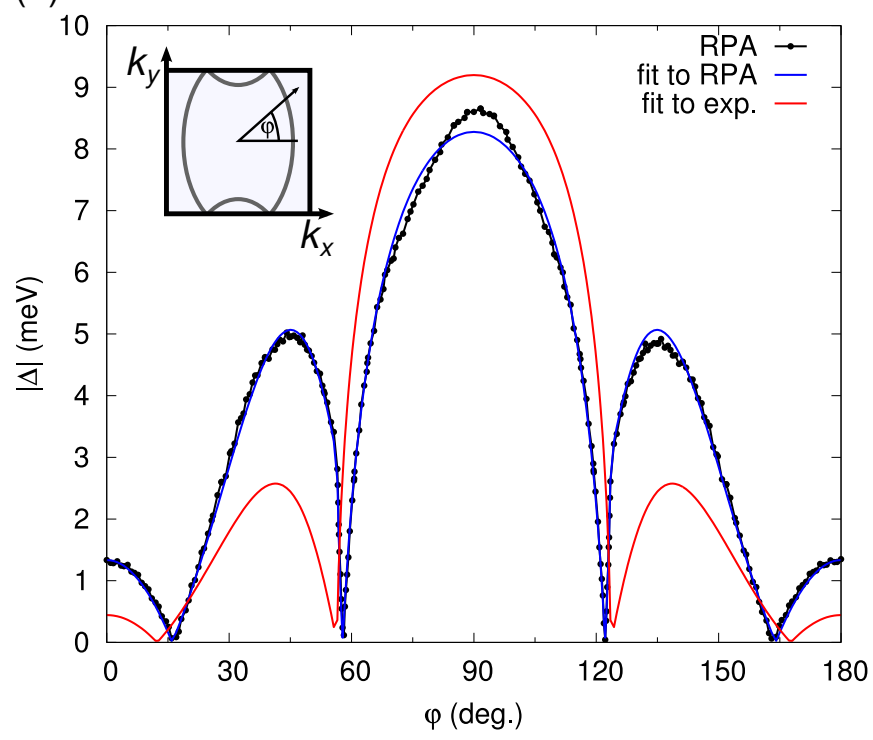

FIG. 11. (a) Polar and (b) linear plot of the magnitude of the superconducting gap $|\Delta|$ on the Fermi surface versus the angle $\varphi$ measured from the $k_{x}$ direction. For the RPA curves and the fit to experiment the energy scale was set to $\Delta_{0}=10 \mathrm{meV}$ and $\Delta_{0}=12.1 \mathrm{meV}$ respectively.

The static spin- and orbital-susceptibilities $\left(\chi^{s, \mathrm{RPA}}\right.$ and $\left.\chi^{c, \mathrm{RPA}}\right)$ are constructed in an RPA framework. Since the interaction term in the Hamiltonian is local and our models are single-orbital in nature, we can restrict the calculation to the diagonal elements of the susceptibility and use scalar equations for the RPA-enhanced susceptibilities.

$$
\chi_{L}^{s, \operatorname{RPA}}(\vec{q})=\frac{\chi_{L}^{0}(\vec{q})}{1-U \chi_{L}^{0}(\vec{q})}, \chi_{L}^{c, \operatorname{RPA}}(\vec{q})=\frac{\chi_{L}^{0}(\vec{q})}{1+U \chi_{L}^{0}(\vec{q})}
$$

Here, $\chi_{L}$ with $L=\{l l l l\}$ denotes the diagonal element of the susceptibility tensor associated with an BEDTTTF site indexed by $l$. The total spin susceptibility is given by the sum over all site-resolved contributions $\chi^{s}(\vec{q})=\frac{1}{2} \sum_{L} \chi_{L}^{s, \operatorname{RPA}}(\vec{q})$.

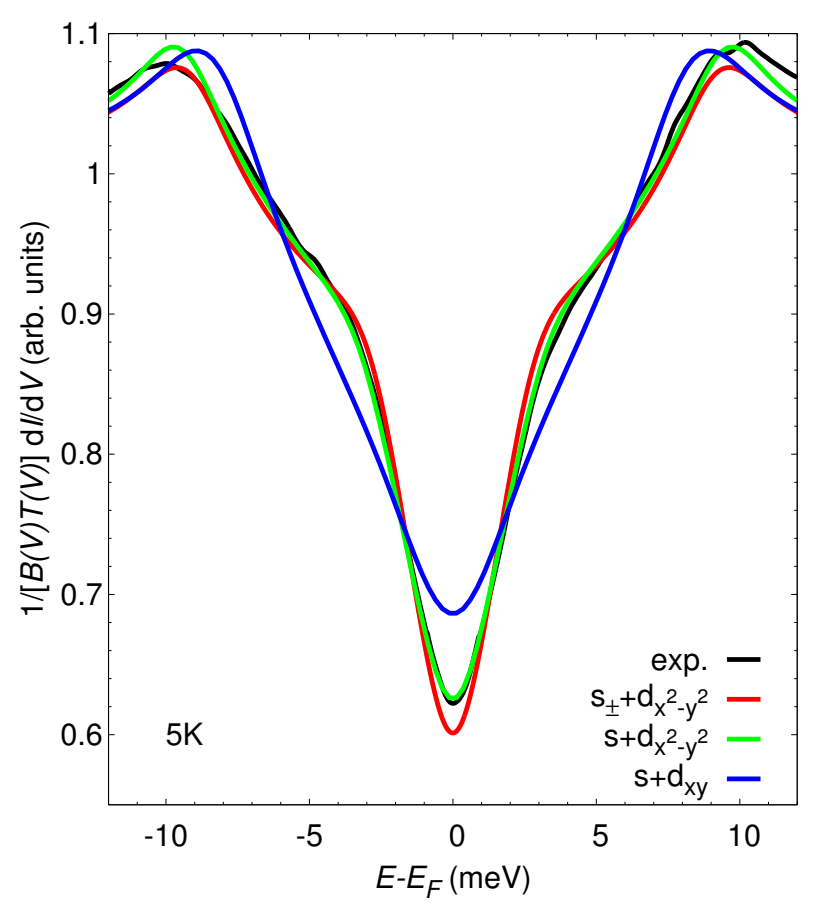

FIG. 12. Experimental spectrum at $5 \mathrm{~K}$ and simulation presented in the main paper $\left(s_{ \pm}+d_{x^{2}-y^{2}}\right)$ compared to simulated spectra of plain $s$-wave combined with $d_{x y}$ and $d_{x^{2}-y^{2}}$ expressions.

The pairing vertex in orbital space for the singlet channel can be calculated using the fluctuation exchange approximation $^{19,20}$.

$$
\begin{aligned}
\Gamma_{s p q t}\left(\vec{k}, \vec{k}^{\prime}\right)= & {\left[\frac{1}{2} U \chi^{s, \mathrm{RPA}}\left(\vec{k}-\vec{k}^{\prime}\right) U+U \chi^{s, \mathrm{RPA}}\left(\vec{k}+\vec{k}^{\prime}\right) U\right.} \\
& \left.-\frac{1}{2} U \chi^{c, \mathrm{RPA}}\left(\vec{k}-\vec{k}^{\prime}\right) U+U\right]_{s p q t}
\end{aligned}
$$

Momenta $\vec{k}$ and $\vec{k}^{\prime}$ are restricted to the Fermi surface. As vectors $\vec{k} \pm \vec{k}^{\prime}$ do not necessarily lie on the grid used in the calculation of the susceptibility $\chi^{0}(\vec{q})$, we use linear interpolation of the grid data.

The pairing vertex in orbital space is transformed into band space using the matrix elements $a_{\mu}^{l}(\vec{k})$.

$$
\begin{gathered}
\tilde{\Gamma}_{\mu \nu}\left(\vec{k}, \vec{k}^{\prime}\right)=\operatorname{Re} \sum_{s p q t} a_{\mu}^{t, *}(\vec{k}) a_{\mu}^{p, *}(-\vec{k})\left[\Gamma_{s p q t}\left(\vec{k}, \vec{k}^{\prime}\right)\right] \\
\times a_{\nu}^{s}\left(\vec{k}^{\prime}\right) a_{\nu}^{q}\left(-\vec{k}^{\prime}\right)
\end{gathered}
$$

Finally, we solve the linearized gap equation by performing an eigendecomposition on the kernel and obtain the dimensionless pairing strength $\lambda_{i}$ and the symmetry function $g_{i}(\vec{k})$.

$$
-\sum_{\nu} \oint_{C_{\nu}} \frac{d k_{\|}^{\prime}}{2 \pi} \frac{1}{2 \pi v_{F}\left(\vec{k}^{\prime}\right)}\left[\Gamma_{\mu \nu}\left(\vec{k}, \vec{k}^{\prime}\right)\right] g_{i}\left(\vec{k}^{\prime}\right)=\lambda_{i} g_{i}(\vec{k})
$$



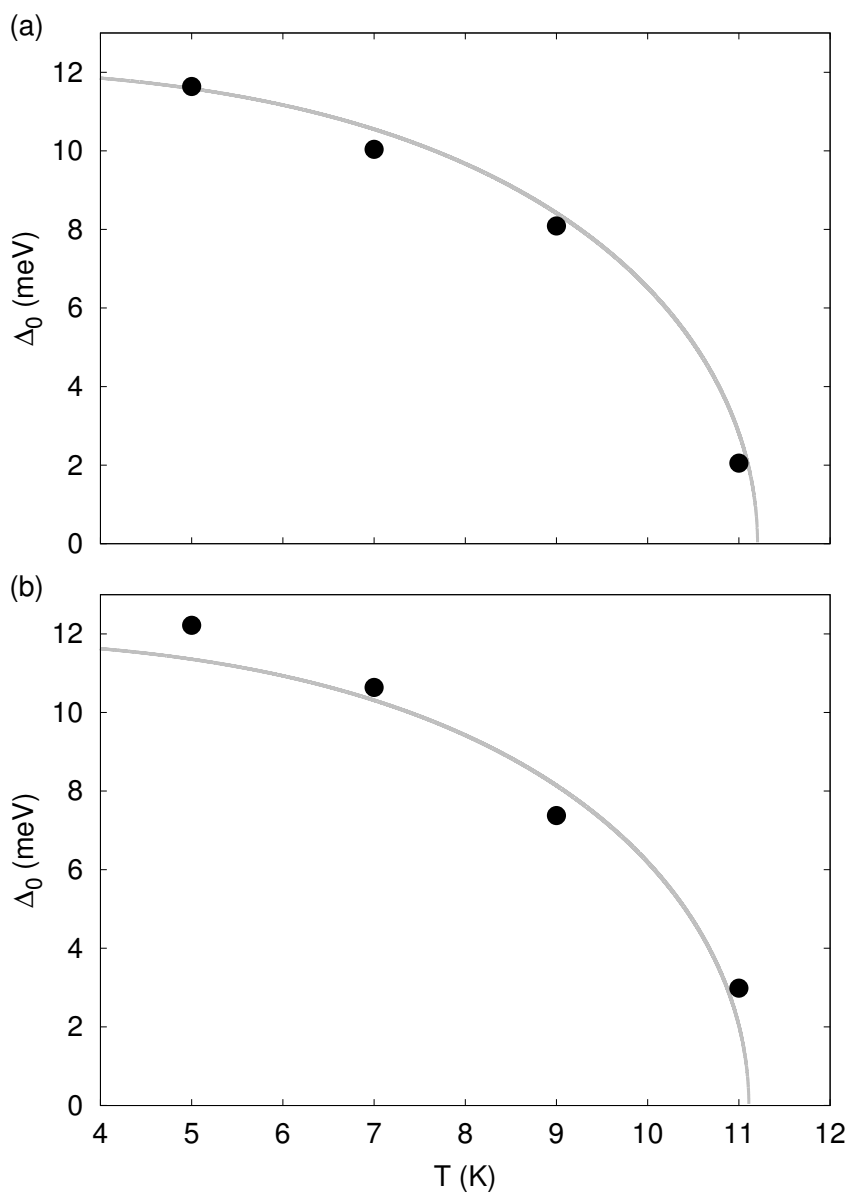

FIG. 13. Fit of the interpolation function for the solution of the s-wave BCS self consistency equation to the temperature dependent gap values obtained from the STS analysis. (a) shows the gaps extracted from the fit using prefactors $c_{i}$ fixed to the values determined from the microscopic calculation, while (b) shows the gaps extracted from a fit to the STS experiment, where also the $c_{i}$ were used as parameters.
In the gap equation we use the singlet symmetrized ver$\operatorname{tex} \Gamma_{\mu \nu}\left(\vec{k}, \vec{k}^{\prime}\right)=\frac{1}{2}\left[\tilde{\Gamma}_{\mu \nu}\left(\vec{k}, \vec{k}^{\prime}\right)+\tilde{\Gamma}_{\mu \nu}\left(\vec{k},-\vec{k}^{\prime}\right)\right]$. The integration runs over the discretized Fermi surface and $v_{F}(\vec{k})$ is the magnitude of the Fermi velocity.

\section{THEORY: RPA SPIN-FLUCTUATION PAIRING, COMPUTATIONAL DETAILS}

For the intra-molecular Coulomb interaction we use a value of $U=0.75 \mathrm{eV}$. The non-interacting susceptibility is calculated on a $50 \times 50 \mathrm{k}$-point grid at an inverse temperature of $\beta=40 \mathrm{eV}^{-1}$. For the solution of the gap equation we used 548 points on the two-dimensional Fermi surface.
* elmers@uni-mainz.de

1 V. A. Ukraintsev, Data evaluation technique for electrontunneling spectroscopy, Phys. Rev. B 53, 11176 (1996).

${ }^{2}$ H. Shinaoka, and M. Imada, Single-Particle Excitations under Coexisting Electron Correlation and Disorder: A Numerical Study of the Anderson-Hubbard Model, J. Phys. Soc. Jpn. 78, 094708 (2009).

${ }^{3}$ S. Diehl, T. Methfessel, U. Tutsch, J. Müller, M. Lang, M. Huth, M. Jourdan, and H.-J. Elmers, Disorder-induced gap in the normal density of states of the organic superconductor $\kappa-(B E D T-T T F)_{2} C u\left[N(C N)_{2}\right] B r$, J. Phys.: Condens. Matter 27, 265601 (2015).

4 A. Savitzky and M. J. E. Golay, Smoothing and Differentiation of Data by Simplified Least Squares Procedures, Anal. Chem. 36, 1627 (1964).

${ }^{5}$ O. J. Taylor, A. Carrington, and J. A. Schlueter, Superconductor-insulator phase separation induced by rapid cooling of $\kappa-(E T)_{2} \mathrm{Cu}\left[\mathrm{N}(\mathrm{CN})_{2}\right] \mathrm{Br}$, Phys. Rev. B 77, 060503(R) (2008).

${ }^{6}$ H. Padamsee, J. E. Neighbor, and C. A. Shiffman, Quasiparticle phenomenology for thermodynamics of strongcoupling superconductors, J. Low Temp. Phys. 12, 387 (1973).

7 O. J. Taylor, A. Carrington, and J. A. Schlueter, Specific-Heat Measurements of the Gap Structure of the Organic Superconductors $\kappa-(E T)_{2} \mathrm{Cu}\left[\mathrm{N}(\mathrm{CN})_{2}\right] \mathrm{Br}$ and $\kappa$ $(E T)_{2} \mathrm{Cu}(\mathrm{NCS})_{2}$, Phys. Rev. Lett. 99, 057001 (2007).

${ }^{8}$ K. Fujita, I. Grigorenko, J. Lee, J. X. Zhu, J. C. Davis, H. Eisaki, S. Uchida, and A. V. Balatsky, Bogoliubov angle and visualisation of particle-hole mixtures in superconductors, Phys. Rev. B 78, 054510 (2008).

9 P. E. Blöchl, O. Jepsen, and O. K. Andersen, Improved tetrahedron method for Brillouin-zone integrations, Phys. Rev. B 49, 16223 (1994). 
10 R. C. Dynes, V. Narayanamurti, and J. P. Garno, Direct Measurement of Quasiparticle-Lifetime Broadening in a Strong-Coupled Superconductor, Phys. Rev. Lett. 41, 1509 (1978).

11 H. Elsinger, J. Wosnitza, S. Wanka, J. Hagel, D. Schweitzer, and W. Strunz, $\kappa$-(BEDTTTF $)_{2} \mathrm{Cu}\left[\mathrm{N}(\mathrm{CN})_{2}\right] \mathrm{Br}:$ A Fully Gapped Strong-Coupling Superconductor, Phys. Rev. Lett. 84, 6098 (2000).

12 U. Geiser, A. J. Schultz, H. W. Wang, D. M. Watkins, D. L. Stupka, J. M. Williams, J. E. Schirber, D. L. Overmeyer, D. Jung, J. J. Novoa, and M.-H. Whangbo, Strain index, lattice softness and superconductivity of organic donor-molecule salts, Crystal and electronic structures of three isostructural salts $\kappa-(B E D T-T T F)_{2} \mathrm{Cu}\left[\mathrm{N}(\mathrm{CN})_{2}\right] \mathrm{X}(\mathrm{X}$ $=C l, B r, I)$, Physica C 174, 475 (1990).

13 D. Guterding, R. Valentí, H. O. Jeschke, Influence of molecular conformations on the electronic structure of organic charge transfer salts, Phys. Rev. B 92, 081109(R) (2015).

14 J. P. Perdew, K. Burke, and M. Ernzerhof, Generalized Gradient Approximation Made Simple, Phys. Rev. Lett. 77, 3865 (1996).
15 E. Yusuf, B. J. Powell, and R. H. McKenzie, Antiferromagnetic spin fluctuations in the metallic phase of quasi-twodimensional organic superconductors, Phys. Rev. B 75, 214515 (2007).

16 S. Graser, T. A. Maier, P. J. Hirschfeld, and D. J. Scalapino, Near-degeneracy of several pairing channels in multiorbital models for the Fe pnictides, New J. Phys. 11, 025016 (2009).

17 D. Guterding, H. O. Jeschke, P. J. Hirschfeld, and R. Valentí, Unified picture of the doping dependence of superconducting transition temperatures in alkali metal/ammonia intercalated FeSe, Phys. Rev. B 91, 041112(R) (2015).

18 D. Guterding, S. Backes, H. O. Jeschke, and R. Valentí, Origin of the superconducting state in the collapsed tetragonal phase of $\mathrm{KFe}_{2} A s_{2}$, Phys. Rev. B 91, 140503(R) (2015).

19 N. E. Bickers, D. J. Scalapino, and S. R. White, Conserving Approximations for Strongly Correlated Electron Systems: Bethe-Salpeter Equation and Dynamics for the Two-Dimensional Hubbard Model, Phys. Rev. Lett. 62, 961 (1989).

20 D. J. Scalapino, E. Loh, Jr., and J. E. Hirsch, d-wave pairing near a spin-density-wave instability, Phys. Rev. B 34, 8190 (1986). 